\title{
On the Vinogradov bound in the three primes Goldbach conjecture
}

\author{
by \\ Ming-Chit Liu (Hong Kong) and Tianze Wang (Kaifeng)
}

1. Introduction and the main result. The Three Primes Goldbach Conjecture (3GC), which was posed in 1742 in a letter of C. Goldbach to L. Euler, states that every odd integer $\geq 9$ is a sum of three odd primes. Assuming the Generalized Riemann Hypothesis (GRH), G. H. Hardy and J. E. Littlewood [HL] proved in 1923 the $3 \mathrm{GC}$ for all sufficiently large odd integers. In 1937 I. M. Vinogradov [V] successfully removed the GRH, namely, he showed that there is a positive integer $V$ such that for any odd integer $n \geq V$ (so the above "sufficiently large" condition is still assumed) one has

$$
n=p_{1}+p_{2}+p_{3}
$$

where $p_{j}$ are odd primes. The $V$ can be $3^{3^{15}}\left(=10^{6,846,168.5 \ldots}\right)$. Therefore Vinogradov qualitatively settled the $3 \mathrm{GC}$ and it remains to consider the quantitative part of the $3 \mathrm{GC}$. That is to remove the condition, "sufficiently large" also from the above Hardy-Littlewood result or equivalently to show that the $V$ in the Vinogradov result can be 9 . Although the 3GC is still not completely settled, Vinogradov's qualitative result is no doubt one of the most remarkable results in the 20th century. Because of the significance of Vinogradov's result we call the value of $V$ the Vinogradov bound. Obviously, to accomplish the quantitative part of the $3 \mathrm{GC}$ we should check all odd integers lying between 9 and $V$. Plainly, the above numerical value for $V$ is far from satisfaction and we should lower the value for $V$ considerably until it falls in the range of the capacity of the latest powerful computer. Along this direction in 1956 Borozdkin [B] showed that $V$ can

2000 Mathematics Subject Classification: 11P32, 11P55, $11 \mathrm{D} 04$.

The work is partially supported by Hong Kong Government RG research grant (HKU7221/99P).

Research of T. Z. Wang supported partially by the National Natural Science Foundation of China (Grant No. 19771029). 
be $\exp (\exp (16.038))\left(=10^{4,008,659.9 \ldots}\right)$. The latest known result for $V$ was obtained by J. R. Chen and T. Z. Wang $[\mathrm{CW}]$ in 1989. They showed that $V$ can be

$$
\exp (\exp (11.503))\left(=10^{43,000.5 \cdots}\right) .
$$

The other direction to investigate the quantitative part of the $3 \mathrm{GC}$ is, of course, to check as many odd integers $<V$ as possible. The latest result in this direction was obtained in 1998 by Y. Saouter [S] who showed that each odd integer $\leq 10^{20}$ has an expression as in (1.1).

In 1997, under the GRH, J. M. Deshouillers, G. Effinger, H. te Riele and D. Zinoviev [DERZ] proved that $V$ can be 9 . That is, under the GRH, the $3 \mathrm{GC}$ is now completely settled. These recent numerical developments stimulate a strong desire to lower the known Vinogradov bound 10 13,000 unconditionally, and to remove the GRH eventually in the quantitative part of the 3GC. In this paper we can lower the value of $V$ further without assuming the GRH. We can prove

Theorem 1. Every odd integer $\geq V=e^{3,100}\left(=10^{1,346.3 \ldots}\right)$ is a sum of three odd primes as in (1.1).

The framework of our proof is based on the Hardy-Littlewood Circle Method. One of the features of the Circle Method is that it leads to asymptotic results and so it works well if some parameters are large enough. Therefore the "sufficiently large" condition is essential and crucial in many steps of the Circle Method. Our goal in Theorem 1 is to replace the "sufficiently large" condition by explicit values of the large parameters. So during the proof there is absolutely no shelter for the "sufficiently large" condition to prevent from being numerically checked.

Besides using some tricks together with the help of computer to obtain better numerical constants in many inequalities, we have mainly the following three differences from the previous work on the Vinogradov bound.

(i) We shall dissect the interval with unit length into four disjoint subsets $\mathcal{M}_{j}$ defined as in (3.4)-(3.7). In order to obtain "smaller values" for the above mentioned essential parameters we choose suitably shorter intervals than the usual major arcs in the Circle Method. Unlike the traditional treatments in the Circle Method where our $\mathcal{M}_{1} \cup \mathcal{M}_{2}$ was regarded as major arcs while our $\mathcal{M}_{3} \cup \mathcal{M}_{4}$ was minor arcs, we refine the method and treat $\mathcal{M}_{2}$ also as minor arcs. We separate $\mathcal{M}_{2}$ from $\mathcal{M}_{1} \cup \mathcal{M}_{2}$ to gain a much more desirable lower bound for $I_{1}(N)$ (defined as in (3.11)) over our major arcs $\mathcal{M}_{1}$. We split $\mathcal{M}_{3} \cup \mathcal{M}_{4}$ in order to better use our new version (Proposition 6.1) of Vinogradov's estimate on minor arcs $\mathcal{M}_{4}$. With the help of Lemmas 5.1 and 5.2 we can obtain good upper bounds for $I_{j}(N)$ over our new "minor arcs" $\mathcal{M}_{j}, j=2,3$. 
(ii) We use [LW, Theorem 8], a new numerical version of the formula for

$$
\psi(t, \chi)=\sum_{n \leq t} \Lambda(n) \chi(n)
$$

(for example, see (3.16) below).

(iii) We obtain as in Proposition 6.1 a new numerical version of the Vinogradov estimate for trigonometric sums over primes which could be useful in most numerical problems whenever the Circle Method is applied.

The material of this paper is arranged as follows. In Section 2, based on the results in [LW, Sections 2 and 3], we establish two explicit double sum estimates in Lemmas 2.1 and 2.2, which play an important role in giving a lower bound for the integral $I_{1}(N)$ on the major arcs $\mathcal{M}_{1}$. Section 3 forms the framework of our proof for Theorem 1 . We construct the four subsets $\mathcal{M}_{j}$ as mentioned above in (i). In Section 4, based on the preparations in Section 2, we give a desirable explicit lower bound for $I_{1}(N)$ in Lemma 4.3. In Section 5, once again by the results of [LW, Sections 2 and 3], we obtain explicit upper bounds for $S(\alpha)$ over the "minor arcs" $\mathcal{M}_{j}, j=2,3$, in Lemmas 5.1 and 5.2. Finally, in Section 6, by Proposition 6.1 we get an explicit estimate for the integral $I_{4}(N)$ over the minor $\operatorname{arcs} \mathcal{M}_{4}$, and then complete the proof of our Theorem 1 .

2. Explicit double sum estimates. Throughout this paper, we use $\chi$ and $\chi_{0}$ to denote a Dirichlet character and a principal character respectively. We use $L(s, \chi)$ to denote Dirichlet $L$-functions. In this section, we give some explicit upper bound estimates for the double sums $\sum_{1}$ and $\sum_{2}$ defined as in Lemmas 2.1 and 2.2 below respectively. The estimates are based on the numerical results given in [LW, Sections 2 and 3], and will be used in Sections 4 and 5. From now on, we always assume $q$ is a positive integer, $N$ is an integer satisfying $N \geq \exp (3100)$, and put

$$
\mathcal{L}:=\log N, \quad P:=\mathcal{L}^{3}, \quad P_{1}:=\mathcal{L}^{6}, \quad T:=\mathcal{L}^{15}, \quad \omega:=3.36 P / q .
$$

Lemma 2.1. For any integer $q$ with $1 \leq q \leq P=\mathcal{L}^{3}$, if $N \geq \exp (3100)$, then

$$
\begin{aligned}
\sum_{1} & :=\sum_{\chi(\bmod q)} \sum_{|\gamma| \leq \omega}^{\prime}\left(1-0.001^{\beta}\right) \beta^{-1} N^{\beta-1} \\
& \leq \begin{cases}0.0194 \mathcal{L}^{-1} & \text { if } \widetilde{\beta} \text { does not exist }, \\
8.2 \cdot 10^{-10} \mathcal{L}^{-1} & \text { if } \widetilde{\beta} \text { exists },\end{cases}
\end{aligned}
$$

where' indicates that the sum $\sum_{|\gamma| \leq \omega}^{\prime}$ is over all the zeros $\varrho=\beta+i \gamma$ of $L(s, \chi)$ satisfying $\beta \geq 1 / 2$ and $|\gamma| \leq \omega$ excluding the possible Siegel zero $\widetilde{\beta}$ in [LW, Lemma 2.1] with $x=P$. 
Proof. In view of

$$
\frac{d}{d \alpha}\left(\frac{1-0.001^{\alpha}}{\alpha}\right)<0 \quad \text { for } 1 / 2 \leq \alpha \leq 1,
$$

we have

$$
\begin{aligned}
\sum_{1} \leq & 2\left(1-0.001^{1 / 2}\right) N^{-1 / 2} N(1 / 2, q, \omega) \\
& +\left\{\int_{1 / 2}^{1-0.478 / \ell(P)}+\int_{1-0.478 / \ell(P)}^{1}\right\} \\
& \times N(\alpha, q, \omega) N^{\alpha-1}(\log N)\left(1-0.001^{\alpha}\right) \alpha^{-1} d \alpha,
\end{aligned}
$$

where $N(\alpha, q, \omega)$ is defined as in [LW, (3.3)]. Here and later on we put $\ell(P):=\log (3.36 P)$. From $(2.1)$ we have $\omega \geq 3.36$ since $q \leq P$. Also by [LW, Theorem 5] we have, for any $y \geq 3.36$,

$$
N\left(1 / 2, \chi_{0}, y\right) \leq(y / \pi) \log y-0.833 y+9.0101 \log y+56 ;
$$

and by [LW, Theorem 6 ] we get, for $y \geq 3.36$ and nonprincipal $\chi$,

$$
N(1 / 2, \chi, y) \leq(y / \pi) \log q y-0.874 y+6.8423 \log q y+15 .
$$

The combination of (2.3) and (2.4) with $y=\omega$ gives, for $1 / 2 \leq \alpha<1$,

$$
\begin{aligned}
& N(\alpha, q, \omega) \\
& \leq(3.36 P / \pi) \log (3.36 P)-0.874 \cdot 3.36 P \\
& \quad+\{6.8423 q \log (3.36 P)+15 q+9.0101 \log (3.36 P / q)\} \\
& \quad+\{-(3.36 / \pi) \log q+0.041 \cdot 3.36\} P / q-6.8423 \log (3.36 P)+41 .
\end{aligned}
$$

The expression in the first curly brackets on the right hand side of $(2.5)$ is clearly increasing with respect to $q$. So for $2 \leq q \leq P,(2.5)$ can be estimated as

$$
\leq 8.82 P \log P .
$$

Again in view of (2.3) one can see easily that (2.6) is also true for $q=1$. Thus the sum of the first term and the first integral on the right hand side of $(2.2)$ is

$$
\begin{aligned}
\leq & (8.82 P \log P)\left\{2\left(1-0.001^{1 / 2}\right) N^{-1 / 2}\right. \\
& \left.+\int_{1 / 2}^{1-0.478 / \ell(P)} N^{\alpha-1}(\log N)\left(1-0.001^{\alpha}\right) \alpha^{-1} d \alpha\right\} .
\end{aligned}
$$

Note that $\left(1-0.001^{\alpha}\right) / \alpha$ is decreasing and $1-0.478 / \log (3.36 P)>1-1 / 50$ since $P=\mathcal{L}^{3} \geq 3100^{3}$. Thus the expression in the last curly brackets in $(2.7)$ is 


$$
\begin{aligned}
& \leq 2\left(1-0.001^{1 / 2}\right) N^{-1 / 2}+2\left(1-0.001^{1 / 2}\right) \int_{1 / 2}^{49 / 50} N^{\alpha-1} \log N d \alpha \\
& \quad+\frac{1-0.001^{49 / 50}}{49 / 50} \int_{49 / 50}^{1-0.478 / \ell(P)} N^{\alpha-1} \log N d \alpha \\
& \leq 0.9176 N^{-1 / 50}+1.0193 N^{-0.478 / \ell(P)} .
\end{aligned}
$$

Hence (2.7) can be estimated further as, for $\mathcal{L} \geq 3100$,

$$
\leq 8.1 \cdot 10^{-10} \mathcal{L}^{-1}
$$

Now we consider two cases according as the Siegel zero $\widetilde{\beta}$ exists or not to estimate the last integral on the right hand side of (2.2).

(i) The $\widetilde{\beta}$ exists. Note that we have

$$
\widetilde{\beta} \geq 1-1 /(9.645908801 \log P) \geq 1-0.11 / \log (3.36 P) .
$$

Also we may use the numerical results in [LW, Sections 2 and 3] with $3.36 P$ instead of $z$ there since $3.36 P \geq 3.36 \cdot 3100^{3}>10^{11}$. By $(2.9)$ and the third row in [LW, Table 1], we see that $N(\alpha, q, \omega)=0$ for $\alpha \geq$ $1-0.3221 / \log (3.36 P)$. Thus in view of the bounds for $\lambda$ in [LW, Tables 4 and 5], we may write the last integral in (2.2) as

$$
\begin{aligned}
& \leq\left\{\int_{1-0.478 / \ell(P)}^{1-0.475 / \ell(P)}+\int_{1-0.475 / \ell(P)}^{1-0.47 / \ell(P)}+\int_{1-0.47 / \ell(P)}^{1-0.46 / \ell(P)}+\int_{1-0.46 / \ell(P)}^{1-0.45 / \ell(P)}\right. \\
& 1-0.42 / \ell(P) \quad 1-0.39 / \ell(P) \quad 1-0.36 / \ell(P) \quad 1-0.33 / \ell(P) \\
& +\int_{1-0.45 / \ell(P)}+\int_{1-0.42 / \ell(P)}+\int_{1-0.39 / \ell(P)}+\int_{1-0.36 / \ell(P)} \\
& 1-0.32 / \ell(P) \\
& \left.+\int_{1-0.33 / \ell(P)}\right\} N(\alpha, q, \omega) N^{\alpha-1}(\log N)\left(1-0.001^{\alpha}\right) \alpha^{-1} d \alpha \text {. }
\end{aligned}
$$

Note that by $(2.1)$ we have $1-0.478 / \log q \omega \geq 0.98$ and consequently $\left(1-0.001^{\alpha}\right) / \alpha \leq\left(1-0.001^{0.98}\right) / 0.98$. Thus in view of the bound $7000 \cdot 2$ of [LW, Table 5], the first integral in (2.10) can be estimated as

$$
\begin{aligned}
& \leq \frac{14000\left(1-0.001^{0.98}\right)}{0.98}\left(N^{-0.475 / \ell(P)}-N^{-0.478 / \ell(P)}\right) \\
& \leq 9 \cdot 10^{7}(\exp (-58.1339)-\exp (-58.5011)) \mathcal{L}^{-1} \\
& \leq 2 \cdot 10^{-18} \mathcal{L}^{-1} .
\end{aligned}
$$


Similarly, the bounds for $N=N(\alpha, q, \omega)$ in [LW, Tables 4 and 5] yield

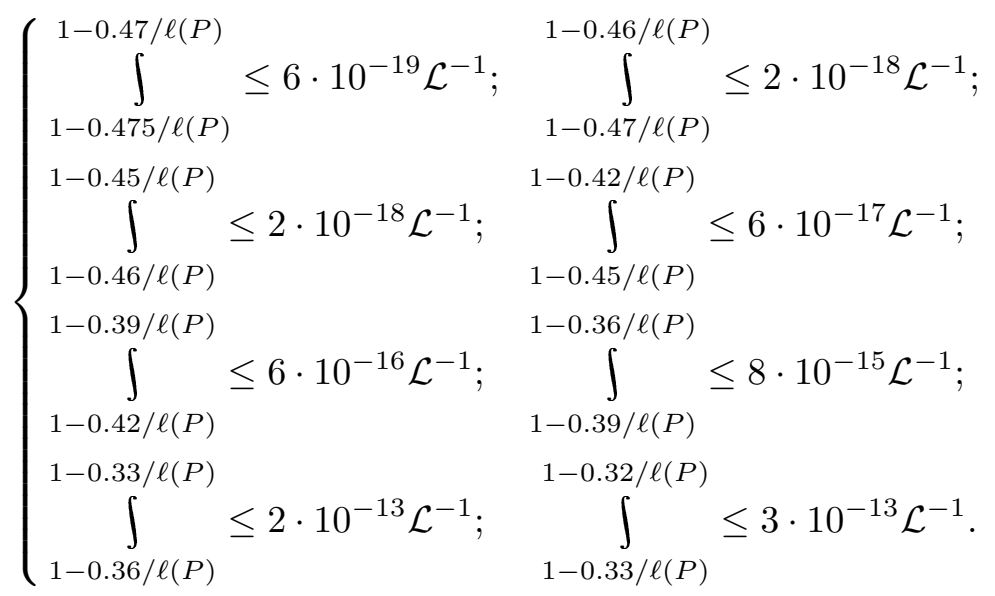

Therefore (2.10), or the last integral in (2.2), satisfies

$$
\int_{1-0.478 / \ell(P)}^{1} \leq 6 \cdot 10^{-13} \mathcal{L}^{-1}
$$

(ii) The Siegel zero $\widetilde{\beta}$ does not exist; that is to say (see [LW, Lemma $2.1])$, there is no zero of the function $\Pi(s)$ defined by [LW, (2.2)] in the region $\sigma \geq 1-1 /\left(c_{1} \log P\right),|t| \leq P / q$, where $c_{1}=9.645908801$. However, in general, it would be possible that $\Pi(s)$ has complex zeros in the region

$$
\sigma \geq 1-1 /\left(c_{1} \log P\right), \quad|t| \leq \omega
$$

since $\omega>P / q$ by (2.1). If there is indeed a zero $\varrho_{1}=\beta_{1}+i \gamma_{1}$ of $\Pi(s)$ in (2.14), then similar to $(2.9)$ we have $\beta_{1} \geq 1-1 /\left(c_{1} \log P\right) \geq 1-0.11 / \ell(P)$. Thus for any zero $\varrho=\beta+i \gamma \neq \varrho_{1}, \bar{\varrho}_{1}$ of $\Pi(s)$ with $|\gamma| \leq \omega$ we have by [LW, Table 1] (for the case $\lambda_{1} \leq 0.12$ and $\lambda_{2}>0.3221$ ), $\beta \leq 1-0.3221 / \ell(P)$; and for $\varrho_{1}$ itself we have by [LW, Lemma 2.1], $\beta_{1} \leq 1-1 /\left(c_{1} \log q \omega\right) \leq$ $1-1 /\left(c_{1} \ell(P)\right)$. So if we use the bound on the right hand side of $(2.13)$, the last integral in $(2.2)$ can be estimated as

$$
\leq 6 \cdot 10^{-13} \mathcal{L}^{-1}+\int_{1-0.32 / \ell(P)}^{1-1 /\left(c_{1} \ell(P)\right)} 2 N^{\alpha-1}(\log N)\left(1-0.001^{\alpha}\right) \alpha^{-1} d \alpha .
$$

In view of $1-0.32 / \ell(P) \geq 1-0.32 / \log \left(3.36 \cdot 3100^{3}\right) \geq 0.987$, for $\mathcal{L} \geq 3100$ the above is

$$
\begin{aligned}
& \leq 6 \cdot 10^{-13} \mathcal{L}^{-1}+\frac{2\left(1-0.001^{0.987}\right)}{0.987}\left(N^{-1 /\left(c_{1} \ell(P)\right)}-N^{-0.32 / \ell(P)}\right) \\
& \leq 0.01938 \mathcal{L}^{-1} .
\end{aligned}
$$


If the above $\varrho_{1}$ does not exist, then by the bounds in (2.11) and (2.12), the last integral in (2.2) can be written as

$$
\begin{aligned}
& \leq 3 \cdot 10^{-13} \mathcal{L}^{-1} \\
& \quad+\int_{1-0.33 / \ell(P)}^{1-1 /\left(c_{1} \log P\right)} N(\alpha, q, \omega) N^{\alpha-1}(\log N)\left(1-0.001^{\alpha}\right) \alpha^{-1} d \alpha .
\end{aligned}
$$

In view of $1-0.33 / \ell(P) \geq 1-0.33 / \ell\left(3100^{3}\right) \geq 0.9869$, we have

$$
\left(1-0.001^{\alpha}\right) / \alpha \leq\left(1-0.001^{0.9869}\right) / 0.9869 \leq 1.0122 .
$$

Hence if we let $K_{1}$ denote the last integral in (2.16), then we can proceed as follows. By [LW, Theorem 2 with $x=3.36 P$ ] we see that the function $\Pi(s)$ has at most two zeros

$$
\varrho^{\prime}=1-\lambda^{\prime} / \ell(P)+i \gamma^{\prime} \text { and } \bar{\varrho}^{\prime}
$$

with $\lambda^{\prime} \leq 0.2067$ and $\left|\gamma^{\prime}\right| \leq \omega$; and we may use the bounds in [LW, Table 1] where the $\lambda^{\prime}$ plays the role of $\lambda_{1}$. If the $\varrho^{\prime}$ in (2.17) exists and satisfies $\lambda^{\prime} \leq 0.12$, then the row with $\lambda_{1} \leq 0.12$ and $\lambda_{2} \geq 0.3221$ in [LW, Table 1] shows that there are only the two zeros $\varrho^{\prime}$ and $\bar{\varrho}^{\prime}$ of $\Pi(s)$ in the region Re $s \geq$ $1-0.3221 / \ell(P),|\operatorname{Im} s| \leq \omega$. Thus $N(\alpha, q, \omega) \leq 2$ for $\alpha \geq 1-0.3221 / \ell(P)$. Consequently by [LW, Table 4 with $\lambda \leq 0.33$ ] we get

$$
\begin{aligned}
K_{1} & \leq \int_{1-0.33 / \ell(P)}^{1-0.3221 / \ell(P)}+\int_{1-0.3221 / \ell(P)}^{1-1 /\left(c_{1} \log P\right)} \\
& \leq 1.0122\left(2 \exp \left(-\mathcal{L} /\left(c_{1} \log P\right)\right)+11 \exp (-0.3221 \mathcal{L} / \ell(P))\right. \\
& \leq 1.0122 \cdot 3100 \mathcal{L}^{-1}\left(3.3 \cdot 10^{-6}+11 \exp (-0.33 \mathcal{L} / \ell(P))\right) \\
& \leq 0.0104 \mathcal{L}^{-1} .
\end{aligned}
$$

If the $\varrho^{\prime}$ in (2.17) exists and satisfies $0.12<\lambda^{\prime} \leq 0.15$, then by the relevant bounds in [LW, Tables 1 and 4$]$ we get

$$
K_{1} \leq \int_{1-0.33 / \ell(P)}^{1-0.2743 / \ell(P)}+\int_{1-0.2743 / \ell(P)}^{1-0.12 / \ell(P)} \leq 0.0027 \mathcal{L}^{-1} .
$$

If the $\varrho^{\prime}$ in (2.17) exists and satisfies $0.15<\lambda^{\prime} \leq 0.2067$, then by [LW, Theorem 1] and the relevant bounds in [LW, Table 4] we get

$$
K_{1} \leq \int_{1-0.33 / \ell(P)}^{1-0.26213 / \ell(P)}+\int_{1-0.26213 / \ell(P)}^{1-0.2067 / \ell(P)}+\int_{1-0.2067 / \ell(P)}^{1-0.15 / \ell(P)} \leq 0.0001 \mathcal{L}^{-1} .
$$

If the $\varrho^{\prime}$ in $(2.17)$ does not exist, then $K_{1}$ can clearly be dominated by the above bound $0.0001 \mathcal{L}^{-1}$. In summary the case $\lambda^{\prime} \leq 0.12$ is the worst and 
we always have $K_{1} \leq 0.0104 \mathcal{L}^{-1}$; and consequently for $\mathcal{L} \geq 3100,(2.16)$ is $\leq 3 \cdot 10^{-13} \mathcal{L}^{-1}+0.0104 \mathcal{L}^{-1} \leq 0.0105 \mathcal{L}^{-1}$. This in combination with $(2.15)$ ensures that the last integral in $(2.2)$ is $\leq 0.01938 \mathcal{L}^{-1}$ if the $\widetilde{\beta}$ does not exist. This together with (2.2), (2.8) and (2.13) completes the proof of Lemma 2.1.

Lemma 2.2. For any integer $q$ with $1 \leq q \leq P$, if $N \geq \exp (3100)$, then

$$
\sum_{2}:=\sum_{\chi(\bmod q)} \sum_{\omega \leq|\gamma| \leq T}^{\prime} N^{\beta-1}|\gamma|^{-1} \leq 0.0126 q \mathcal{L}^{-4}
$$

where $\omega, P$ and $T$ are defined as in (2.1).

Proof. We have

$$
\begin{aligned}
\sum_{2} & =N^{-1 / 2} \sum_{\chi(\bmod q)} \sum_{\omega \leq|\gamma| \leq T, \beta \geq 1 / 2}^{\prime}|\gamma|^{-1} \\
& +\left\{\int_{1 / 2}^{19 / 20}+\int_{19 / 20}^{1}\right\} N^{\alpha-1}(\log N) \sum_{\chi(\bmod q)} \sum_{\omega \leq|\gamma| \leq T, \beta \geq \alpha}^{\prime}|\gamma|^{-1} d \alpha .
\end{aligned}
$$

For any $\alpha$ with $1 / 2 \leq \alpha<1$, we have, in view of [LW, (3.3)],

$$
\sum_{\chi(\bmod q)} \sum_{\omega \leq|\gamma| \leq T, \beta \geq \alpha}^{\prime}|\gamma|^{-1} \leq T^{-1} N(\alpha, q, T)+\int_{\omega}^{T} y^{-2} N(\alpha, q, y) d y .
$$

Using the bound in (2.4) with $q=P$ and noting $P=\mathcal{L}^{3}, T=\mathcal{L}^{15},(2.19)$ can be estimated as $\leq 51 P \log ^{2} \mathcal{L}$. Hence the sum of the first term and the first integral on the right hand side of (2.18) is, for $N \geq \exp (3100)$,

$$
\begin{aligned}
& \leq 51 N^{-1 / 2} P \log ^{2} \mathcal{L}+51 P(\log \mathcal{L})^{2} \int_{1 / 2}^{19 / 20} N^{\alpha-1} \log N d \alpha \\
& \leq \exp (-90) \mathcal{L}^{-4} .
\end{aligned}
$$

Now we use the bound given by (2.19) to estimate the last integral on the right hand side of (2.18). In view of $q \leq P=\mathcal{L}^{3}$ and $T=\mathcal{L}^{15}$, for $19 / 20 \leq \alpha<1$ we have, by [LW, Theorem 7],

$$
\begin{aligned}
N(\alpha, q, T) \leq & (17102+254231 /(18 \log \mathcal{L}))(18 \log \mathcal{L})^{5.7} \mathcal{L}^{69 / 20} \\
& +16541(15 \log \mathcal{L})^{6} .
\end{aligned}
$$

Hence the total contribution to (2.18) from the first term on the right hand side of (2.19) can be estimated as for $\mathcal{L} \geq 3100$,

$$
\begin{aligned}
\leq & (17102+254231 /(18 \log \mathcal{L}))(18 \log \mathcal{L})^{5.7} \mathcal{L}^{69 / 20-15} \\
& +16541 \mathcal{L}^{-15}(15 \log \mathcal{L})^{6} \\
\leq & 1.8 \cdot 10^{-10} \mathcal{L}^{-4} .
\end{aligned}
$$


Now in view of (2.18) and (2.19), the estimation for (2.18) is reduced to the estimate for

$$
\begin{aligned}
\int_{19 / 20}^{1} N^{\alpha-1} \mathcal{L} \int_{\omega}^{T} y^{-2} N(\alpha, q, y) d y d \alpha & \\
& =\int_{\omega}^{T} y^{-2} \int_{19 / 20}^{1} N^{\alpha-1} \mathcal{L} N(\alpha, q, y) d \alpha d y .
\end{aligned}
$$

If we use [LW, Lemma 2.1] with $x$ there equal to $q y$, the innermost integral on the right hand side of (2.22) may be written as

$$
=\int_{19 / 20}^{1-1 /\left(c_{1} \log q y\right)}=\int_{19 / 20}^{1-0.478 / \log q y}+\int_{1-0.478 / \log q y}^{1-1 /\left(c_{1} \log q y\right)},
$$

where $c_{1}$ is defined as in [LW, Lemma 2.1]. Write $q_{1}=\max \left(10^{5} q^{-1}, 10^{4} \log q\right)$. If $y \geq q_{1}$ then by [LW, Theorem 7], the first integral on the right hand side of (2.23) can be estimated as

$$
\begin{aligned}
\leq & (17102+254231 / \log q y) \frac{(\log q y)^{6} \mathcal{L}}{\log \left(N q^{-3} y^{-4}\right)}\left(N q^{-3} y^{-4}\right)^{-0.478 / \log q y} \\
& +16541(\log y)^{6} N^{-0.478 / \log q y} .
\end{aligned}
$$

If $y \leq q_{1}$ then as the $y$ in $(2.22)$ is $\geq \omega=3.36 P q^{-1}$ we get $3.36 P q^{-1} \leq q_{1}$. This leads to $q \geq 7 \cdot 10^{5}$ on noting $P=\mathcal{L}^{3} \geq 3100^{3}$. Thus $q_{1}=10^{4} \log q$, and it is easy to see that the bound in (2.4) is greater than that in (2.3). So by (2.4) we get

$$
N(\alpha, q, y) \leq \varphi(q)((y / \pi) \log q y-0.874 y+6.8423 \log q y+15) .
$$

Now let $K_{2}$ denote the contribution to (2.22) from the first integral on the right hand side of (2.23). Then we can estimate $K_{2}$ as follows. If $\omega \geq q_{1}$ (so $y \geq q_{1}$ ), then we may use (2.24) to get

$$
\begin{aligned}
K_{2} \leq & q \mathcal{L}^{-4} \int_{\log (3.36 P)}^{\log P T} e^{-y}\left((17102+254231 / y) y^{6} \mathcal{L}^{5}(\mathcal{L}-4 y)^{-1}\right. \\
& \left.\times e^{-0.478(\mathcal{L}-4 y) y^{-1}}+16541 y^{6} \mathcal{L}^{4} e^{-0.478 \mathcal{L} y^{-1}}\right) d y
\end{aligned}
$$

on noting $q \leq P$ and $\omega=3.36 P q^{-1}$ by (2.1). The integral in (2.26), as a function of $\mathcal{L}$, is shown by Mathematica software to take its supremum at $\mathcal{L}=3100$ for $\mathcal{L} \geq 3100$; and the supremum is $\leq 0.00031$. Hence in this case $K_{2} \leq 0.00031 q \mathcal{L}^{-4}$. If $\omega<q_{1}$, we may write the integral $\int_{\omega}^{T}$ in $(2.22)$ as 
$\int_{\omega}^{q_{1}}+\int_{q_{1}}^{T}$. Then by $(2.24)$ and $(2.25)$ we get

$$
\begin{aligned}
K_{2} \leq & 0.00031 q \mathcal{L}^{-4}+q \mathcal{L}^{-4} \int_{\log \left(3.36 \mathcal{L}^{3}\right)}^{\log \left(10^{4} \mathcal{L}^{3} \log \mathcal{L}^{3}\right)} \mathcal{L}^{4}(y / \pi-0.874 \\
& \left.+(6.8423 / 7) 10^{-5} y e^{-y}+(15 / 7) \cdot 10^{-5} e^{-y}\right) e^{-0.478 \mathcal{L} / y} d y .
\end{aligned}
$$

By Mathematica, the last integral in (2.27), as a function of $\mathcal{L}$, takes its supremum at $\mathcal{L}=3100$ for $\mathcal{L} \geq 3100$ and the supremum is $\leq 0.00197$. Thus by $(2.27)$,

$$
K_{2} \leq(0.00031+0.00197) q \mathcal{L}^{-4}=0.00228 q \mathcal{L}^{-4} .
$$

For the last integral in (2.23), if we write $\alpha=1-\lambda / \log q y$, then it can be written as

$$
\frac{\log N}{\log q y} \int_{1 / c_{1}}^{0.478} N^{-\lambda / \log q y} N(1-\lambda / \log q y, q, y) d \lambda .
$$

Note that by (2.1) we have $q y \geq q \omega \geq 3.36 P \geq 10^{11}$. Thus the bounds for $\lambda$ in [LW, Tables 3 to 5] can be applied, and we may use [LW, Theorems 1 and 2] with $x=q y \geq 10^{11}>8 \cdot 10^{9}$. In view of the bounds for $\lambda$ in [LW, Tables 4 and 5], we write $(2.29)$ as

$$
\begin{aligned}
\frac{\log N}{\log q y}\left\{\int_{1 / c_{1}}^{0.36}+\int_{0.36}^{0.39}+\int_{0.39}^{0.42}+\int_{0.42}^{0.45}+\int_{0.45}^{0.46}+\int_{0.46}^{0.47}\right. \\
\left.+\int_{0.47}^{0.475}+\int_{0.475}^{0.478}\right\} N^{-\lambda / \log q y} N(1-\lambda / \log q y, q, y) d \lambda .
\end{aligned}
$$

If we use the relevant bounds in [LW, Tables 4 and 5] to estimate $N(1-$ $\lambda / \log q y, q, y)$ in (2.30), the total contribution to (2.22) from the last seven integrals in (2.30) can be estimated as

$$
\begin{aligned}
\leq & q \mathcal{L}^{-4} \int_{\log (3.36 P)}^{\log P T} \mathcal{L}^{4} e^{-y}\left(35 e^{-0.36 \mathcal{L} / y}+54 e^{-0.39 \mathcal{L} / y}\right. \\
& +93 e^{-0.42 \mathcal{L} / y}+110 e^{-0.45 \mathcal{L} / y}+372 e^{-0.46 \mathcal{L} / y}+1004 e^{-0.47 \mathcal{L} / y} \\
& \left.+12332 e^{-0.475 \mathcal{L} / y}-14000 e^{-0.478 \mathcal{L} / y}\right) d y .
\end{aligned}
$$

By Mathematica, it can be checked that the last integral in (2.31), as a function of $\mathcal{L}$, takes its supremum at $\mathcal{L}=3100$ if $\mathcal{L} \geq 3100$. With $\mathcal{L}=3100$, the integral is $\leq 3.68 \cdot 10^{-13}$, and then $(2.31)$ is

$$
\leq 3.68 \cdot 10^{-13} q \mathcal{L}^{-4} \text {. }
$$


Now we turn to the estimate related to the term

$$
\frac{\log N}{\log q y} \int_{1 / c_{1}}^{0.36}
$$

in (2.30), and let $K_{3}$ denote its contribution to (2.22). Note that by [LW, Theorem 2] the function $\Pi(s)$ defined by [LW, (2.2)] has at most two zeros

$$
\varrho_{1}=1-\lambda_{1} / \log q y+i \gamma_{1} \text { and } \bar{\varrho}_{1}
$$

with $\lambda_{1} \leq 0.2067$ and $\left|\gamma_{1}\right| \leq y$. If $\varrho_{1}$ exists and satisfies $\lambda_{1} \leq 0.12$, then by [LW, Table 1] we know that $\Pi(s)$ has no other zero in the region $\operatorname{Re} s \geq$ $1-0.3221 / \log q y,|\operatorname{Im} s| \leq y$ except for $\varrho_{1}$ and $\varrho_{1}$. Thus we have $N(1-$ $\lambda / \log q y, q, y) \leq 2$ for $\lambda \leq 0.3221$; and as a result, (2.33) is, by the relevant bounds in [LW, Table 4],

$$
\begin{aligned}
& \leq \frac{\log N}{\log q y}\left\{\int_{1 / c_{1}}^{0.3221} 2 N^{-\lambda / \log q y} d \lambda+\int_{0.3221}^{0.33} 13 N^{-\lambda / \log q y} d \lambda\right. \\
& \left.\quad+\int_{0.33}^{0.36} 20 N^{-\lambda / \log q y} d \lambda\right\} \\
& =2 N^{-1 /\left(c_{1} \log q y\right)}+11 N^{-0.3221 / \log q y}+7 N^{-0.33 / \log q y}-20 N^{-0.36 / \log q y} .
\end{aligned}
$$

Hence in this case we have, for $\mathcal{L} \geq 3100$,

$$
\begin{aligned}
K_{3} & \leq \int_{\omega}^{T} y^{-2}\left(2 N^{-1 /\left(c_{1} \log q y\right)}+11 N^{-0.3221 / \log q y}+7 N^{-0.33 / \log q y}\right. \\
& \leq 0.0102654 q \mathcal{L}^{-4}
\end{aligned}
$$

If the $\varrho_{1}$ in $(2.34)$ exists and satisfies $0.12<\lambda_{1} \leq 0.15$, then $N(1-$ $\lambda / \log q y, q, y) \leq 2$ for any $\lambda \leq 0.2743$; so in view of the relevant bounds in [LW, Tables 3 and 4] we get, for $\mathcal{L} \geq 3100$,

$$
\begin{aligned}
K_{3} \leq & q \mathcal{L}^{-4} \int_{\log (3.36 P)}^{\log P T} \mathcal{L}^{4} e^{-y}\left(2 e^{-0.12 \mathcal{L} / y}+6 e^{-0.2743 \mathcal{L} / y}\right. \\
& +e^{-0.28 \mathcal{L} / y}+e^{-0.3 \mathcal{L} / y}+e^{-0.31 \mathcal{L} / y}+2 e^{-0.32 \mathcal{L} / y} \\
& \left.+7 e^{-0.33 \mathcal{L} / y}-20 e^{-0.36 \mathcal{L} / y}\right) d y \\
\leq & 0.0016 q \mathcal{L}^{-4} .
\end{aligned}
$$

If the $\varrho_{1}$ in $(2.34)$ exists and satisfies $0.15<\lambda_{1}$, or if it does not exist, then by [LW, Theorem 1] and the relevant bounds in [LW, Tables 3 and 4], we 
have, for $\mathcal{L} \geq 3100$,

$$
\begin{aligned}
K_{3} \leq & q \mathcal{L}^{-4} \int_{\log (3.36 P)}^{\log P T} \mathcal{L}^{4} e^{-y}\left(2 e^{-0.15 \mathcal{L} / y}+2 e^{-0.2067 \mathcal{L} / y} 3 e^{-0.26213 \mathcal{L} / y}\right. \\
& +e^{-0.27 \mathcal{L} / y}+e^{-0.28 \mathcal{L} / y}+e^{-0.3 \mathcal{L} / y}+e^{-0.31 \mathcal{L} / y}+2 e^{-0.32 \mathcal{L} / y} \\
& \left.+7 e^{-0.33 \mathcal{L} / y}-20 e^{-0.36 \mathcal{L} / y}\right) d y \\
\leq & 0.00006 q \mathcal{L}^{-4}
\end{aligned}
$$

From (2.18), (2.20), (2.21), (2.28), (2.32) and (2.35) to (2.37), the proof of Lemma 2.2 is complete.

3. The circle method. From now on we let

$$
Q:=N \mathcal{L}^{-7} \text {. }
$$

By Dirichlet's lemma on rational approximations, each $\alpha$ in $[1 / Q, 1+1 / Q]$ may be written as

$$
\begin{aligned}
& \alpha=a / q+\eta \quad \text { with } 1 \leq a \leq q \leq Q \\
&(a, q):=\operatorname{gcd}(a, q)=1,|\eta| \leq 1 /(q Q) .
\end{aligned}
$$

Denote by $\mathcal{M}(a, q)$ the interval centered at $a / q$ with radius $1 /(q Q)$. Then all the $\mathcal{M}(a, q)$ 's with $1 \leq q \leq P_{1}=\mathcal{L}^{6}, 1 \leq a \leq q$ and $(a, q)=1$ are mutually disjoint since $P_{1}<Q / 2$ on noting $\mathcal{L} \geq 3100$. Put, for $1 \leq q \leq P$ $=\mathcal{L}^{3}$

$$
\delta(N, q):=3.36 P /(10 \pi q N),
$$

which is clearly $\leq 1 /(q Q)=\mathcal{L}^{7} /(q N)$. Let

$$
\begin{aligned}
& \mathcal{M}_{1}:=\bigcup_{1 \leq q \leq P} \bigcup_{\substack{1 \leq a \leq q \\
(a, q)=1}}[a / q-\delta(N, q), a / q+\delta(N, q)], \\
& \mathcal{M}_{2}:=\bigcup_{1 \leq q \leq P} \bigcup_{\substack{1 \leq a \leq q \\
(a, q)=1}} \mathcal{M}(a, q)-\mathcal{M}_{1}, \\
& \mathcal{M}_{3}:=\bigcup_{P<q \leq P 1} \bigcup_{\substack{1 \leq a \leq q \\
(a, q)=1}} \mathcal{M}(a, q), \\
& \mathcal{M}_{4}:=[1 / Q, 1+1 / Q]-\bigcup_{1 \leq j \leq 3} \mathcal{M}_{j} .
\end{aligned}
$$

As usual, for any real $\alpha$ we let $e(\alpha):=e^{2 \pi i \alpha}$, and put

$$
S(\alpha):=\sum_{0.001 N \leq n \leq N} \Lambda(n) e(\alpha n) .
$$


Set

$$
I(N):=\sum_{\substack{p_{1}+p_{2}+p_{3}=N \\ 0.001 N \leq p_{j} \leq N, 1 \leq j \leq 3}}\left(\log p_{1}\right)\left(\log p_{2}\right)\left(\log p_{3}\right)
$$

Then

$$
\int_{1 / Q}^{1+1 / Q} S^{3}(\alpha) e(-N \alpha) d \alpha=I(N)+\sum_{\left(p_{1}, p_{2}, p_{3}\right)}\left(\log p_{1}\right)\left(\log p_{2}\right)\left(\log p_{3}\right)
$$

where the sum $\sum_{\left(p_{1}, p_{2}, p_{3}\right)}$ is over all the prime triplets $\left(p_{1}, p_{2}, p_{3}\right)$ satisfying $p_{1}^{l_{1}}+p_{2}^{l_{2}}+p_{3}^{l_{3}}=N$ and $0.001 N \leq p_{j} \leq N$ for $1 \leq j \leq 3$ with at least one of the positive integers $l_{j} \geq 2$ for $1 \leq j \leq 3$. So the sum in (3.10) is $\leq 3 N^{3 / 2} \mathcal{L}^{3}$. For $1 \leq j \leq 4$ put

$$
I_{j}(N):=\int_{\mathcal{M}_{j}} S^{3}(\alpha) e(-N \alpha) d \alpha .
$$

Then by (3.10) we get

$$
I(N) \geq \sum_{1 \leq j \leq 4} I_{j}(N)-3 N^{3 / 2} \mathcal{L}^{3} .
$$

Now we give a transformation for $S(\alpha)$ defined by (3.8) when $\alpha$ is any point in $\mathcal{M}(a, q)$ with $a$ and $q$ satisfying (3.2) and $q \leq P_{1}$. In view of $\alpha=a / q+\eta$ in (3.2), by the orthogonality relation for Dirichlet characters, one can deduce that

$$
S(\alpha)=\frac{1}{\varphi(q)} \sum_{\chi(\bmod q)} G(a, \bar{\chi}) S(\eta, \chi)+(\theta / \log 2) \mathcal{L}^{2}
$$

here and throughout, $\theta$ denotes a complex number with $|\theta| \leq 1$, not necessarily the same at different occurrences, and

$$
\begin{aligned}
G(a, \chi) & :=\sum_{1 \leq l \leq q,(l, q)=1} \chi(l) e(a l / q), \\
S(\eta, \chi) & :=\sum_{0.001 N \leq n \leq N} \Lambda(n) \chi(n) e(n \eta) .
\end{aligned}
$$

From (3.15) and [LW, Theorem 8] we get

$$
S(\eta, \chi)=\delta(\chi) \int_{0.001 N}^{N} e(\eta t) d t-\sum_{\substack{|\gamma| \leq T \\ \beta \geq 1 / 2}} \int_{0.001 N}^{N} t^{\varrho-1} e(\eta t) d t+R_{11}
$$

where

$$
\left|R_{11}\right| \leq(1.3818+4.3367 N|\eta|) N T^{-1} \mathcal{L}^{2}
$$


Substituting (3.16) into (3.13) and in view of $G\left(a, \chi_{0}\right)=\mu(q)$ we get

$$
\begin{aligned}
S(\alpha)= & \frac{\mu(q)}{\varphi(q)} \int_{0.001 N}^{N} e(\eta t) d t \\
& -\frac{1}{\varphi(q)} \sum_{\chi(\bmod q)} G(a, \bar{\chi}) \sum_{\substack{|\gamma| \leq T \\
\beta \geq 1 / 2}} \int_{0.001 N}^{N} t^{\varrho-1} e(\eta t) d t+R_{12},
\end{aligned}
$$

where, by (3.17) and $|G(a, \bar{\chi})| \leq q^{1 / 2}$,

$$
\left|R_{12}\right| \leq(1.3818+4.3367 N|\eta|) N T^{-1} q^{1 / 2} \mathcal{L}^{2}+\mathcal{L}^{2} / \log 2 .
$$

From now on we specify $\widetilde{\beta}$ to denote the fixed possible Siegel zero in [LW, Lemma 2.1] with $x=P=\mathcal{L}^{3}\left(\geq 8 \cdot 10^{9}\right)$, and the corresponding real primitive character and its modulus are denoted by $\tilde{\chi}$ and $\widetilde{r}$ respectively. Note that $987 \leq \widetilde{r} \leq P=\mathcal{L}^{3}$ and

$$
\widetilde{\beta} \geq 1-1 /(9.645908801 \log P) .
$$

Then for the $\alpha$ in (3.2) with $1 \leq q \leq P$ we can write (3.18) further as

$$
\begin{aligned}
S(\alpha)= & \frac{\mu(q)}{\varphi(q)} J(\eta)-\frac{\delta(q)}{\varphi(q)} G\left(a, \widetilde{\chi} \chi_{0}\right) J(\widetilde{\beta}, \eta) \\
& -\frac{1}{\varphi(q)} \sum_{\chi(\bmod q)} G(a, \bar{\chi}) \sum_{|\gamma| \leq T}^{\prime} J(\varrho, \eta)+R_{12} \\
= & : H(a, q, \eta)+R_{12} ;
\end{aligned}
$$

here and from now on, $\delta(q)=1$ if $\widetilde{r} \mid q, \delta(q)=0$ otherwise,

$$
J(\eta):=\int_{0.001 N}^{N} e(\eta t) d t \text { and } J(\varrho, \eta):=\int_{0.001 N}^{N} t^{\varrho-1} e(\eta t) d t,
$$

and the ' indicates that the sum $\sum_{|\gamma| \leq T}^{\prime}$ is over all nontrivial zeros $\varrho=$ $\beta+i \gamma \neq \widetilde{\beta}$ of $L(s, \chi)$ with $\beta \geq 1 / 2$. This is the desired transformation for $S(\alpha)$.

The remainder of this section is devoted to a transformation for $I_{1}(N)$ defined by (3.11). By (3.4) we get

$$
I_{1}(N)=\sum_{1 \leq q \leq P} \sum_{\substack{1 \leq a \leq q \\(a, q)=1}} e(-a N / q) \int_{|\eta| \leq \delta(N, q)} S^{3}(a / q+\eta) e(-N \eta) d \eta .
$$

Note that by (3.19), (3.3), $q \leq P$ and $T=\mathcal{L}^{15}$, the $R_{12}$ in (3.21) can be estimated as, if $\alpha \in \mathcal{M}_{1}$,

$$
\left|R_{12}\right| \leq 0.48 N \mathcal{L}^{-10}
$$


Now if we replace one of $S(a / q+\eta)$ on the right hand side of (3.23) by $H(a, q, \eta)+R_{12}$ in (3.21) then there is an error term due to $R_{12}$; and in view of (3.24) and (3.8), the total error to (3.23) induced by $R_{12}$ has absolute value, by [RS1, Theorem 6$]$,

$$
\begin{aligned}
& \leq 0.48 N \mathcal{L}^{-10} \sum_{0.001 N \leq n \leq N} \Lambda(n)^{2} \leq 0.48 \cdot 1.001102 N^{2} \mathcal{L}^{-9} \\
& \leq 0.481 N^{2} \mathcal{L}^{-9}
\end{aligned}
$$

By (3.21), (3.23) and (3.25) we get

$$
\begin{aligned}
& I_{1}(N)=\sum_{1 \leq q \leq P} \sum_{\substack{1 \leq a \leq q \\
(a, q)=1}} e(-a N / q) \\
& \times \int_{|\eta| \leq \delta(N, q)} H(a, q, \eta)\left(H(a, q, \eta)+R_{12}\right)^{2} e(-N \eta) d \eta+0.481 \theta N^{2} \mathcal{L}^{-9} .
\end{aligned}
$$

Note that by (3.21),

$$
\begin{aligned}
& H(a, q, \eta)\left(H(a, q, \eta)+R_{12}\right)^{2} \\
& \quad=H(a, q, \eta)^{3}+2 S(a / q+\eta)^{2} R_{12}-3 S(a / q+\eta) R_{12}^{2}+R_{12}^{3} .
\end{aligned}
$$

Thus (3.26) can be rewritten as

$$
\stackrel{I_{1}(N)}{=} \sum_{1 \leq q \leq P} \sum_{\substack{1 \leq a \leq q \\(a, q)=1}} e(-a N / q) \int_{|\eta| \leq \delta(N, q)} H^{3}(a, q, \eta) e(-N \eta) d \eta+R_{13},
$$

where by (3.24), (3.25), (3.3) and $\mathcal{L} \geq 3100$,

$$
\left|R_{13}\right| \leq 3 N^{2} \mathcal{L}^{-9} .
$$

By (3.27) and the definition of $H(a, q, \eta)$ in (3.21) we may transform $I_{1}(N)$ as in (3.29) below, which is the desired form for $I_{1}(N)$ :

$$
I_{1}(N)=\sum_{1 \leq q \leq P} \frac{\mu(q)}{\varphi(q)^{3}} \sum_{\substack{1 \leq a \leq q \\(a, q)=1}} e(-a N / q)
$$

$$
\times \int_{|\eta| \leq \delta(N, q)} J(\eta)^{3} e(-N \eta) d \eta-3 \sum_{1 \leq q \leq P} \frac{|\mu(q)|}{\varphi(q)^{3}}
$$

$$
\times \sum_{\chi(\bmod q)} \sum_{\substack{1 \leq a \leq q \\(a, q)=1}} G(a, \bar{\chi}) e(-a N / q) \sum_{|\gamma| \leq T}^{\prime} \int_{|\eta| \leq \delta(N, q)} J(\eta)^{2} J(\varrho, \eta) e(-N \eta) d \eta
$$




$$
\begin{aligned}
& +3 \sum_{1 \leq q \leq P} \frac{\mu(q)}{\varphi(q)^{3}} \sum_{\chi_{1}(\bmod q)} \sum_{\chi_{2}(\bmod q)} \sum_{\substack{1 \leq a \leq q \\
(a, q)=1}} G\left(a, \bar{\chi}_{1}\right) G\left(a, \bar{\chi}_{2}\right) e(-a N / q) \\
& \times \sum_{\left|\gamma_{1}\right| \leq T}^{\prime} \sum_{\left|\gamma_{2}\right| \leq T}^{\prime} \int_{|\eta| \leq \delta(N, q)} J(\eta) J\left(\varrho_{1}, \eta\right) J\left(\varrho_{2}, \eta\right) e(-N \eta) d \eta \\
& -\sum_{1 \leq q \leq P} \frac{1}{\varphi(q)^{3}} \sum_{\chi_{1}(\bmod q)} \sum_{\chi_{2}(\bmod q)} \sum_{\chi_{3}(\bmod q)} \sum_{\substack{1 \leq a \leq q \\
(a, q)=1}} \prod_{1 \leq j \leq 3} G\left(a, \bar{\chi}_{j}\right) e(-a N / q)
\end{aligned}
$$$$
\times \sum_{\left|\gamma_{1}\right| \leq T}^{\prime} \sum_{\left|\gamma_{2}\right| \leq T}^{\prime} \sum_{\left|\gamma_{3}\right| \leq T}^{\prime} \int_{|\eta| \leq \delta(N, q)} e(-N \eta) \prod_{1 \leq j \leq 3} J\left(\varrho_{j}, \eta\right) d \eta
$$$$
-3 \widetilde{E} \sum_{\substack{1 \leq q \leq P \\ \widetilde{r} \mid q}} \frac{|\mu(q)|}{\varphi(q)^{3}} \sum_{\substack{1 \leq a \leq q \\(a, q)=1}} G\left(a, \tilde{\chi} \chi_{0}\right) e(-a N / q)
$$$$
\times \int_{|\eta| \leq \delta(N, q)} J(\eta)^{2} J(\widetilde{\beta}, \eta) e(-N \eta) d \eta
$$$$
+6 \widetilde{E} \sum_{\substack{1 \leq q \leq P \\ \widetilde{r} \mid q}} \frac{\mu(q)}{\varphi(q)^{3}} \sum_{\chi(\bmod q)} \sum_{\substack{1 \leq a \leq q \\(a, q)=1}} G(a, \bar{\chi}) G\left(a, \tilde{\chi} \chi_{0}\right) e(-a N / q)
$$$$
\times \sum_{|\gamma| \leq T}^{\prime} \int_{|\eta| \leq \delta(N, q)} J(\eta) J(\widetilde{\beta}, \eta) J(\varrho, \eta) e(-N \eta) d \eta
$$$$
-3 \widetilde{E} \sum_{\substack{1 \leq q \leq P \\ \widetilde{r} \mid q}} \frac{1}{\varphi(q)^{3}} \sum_{\chi_{1}(\bmod q)} \sum_{\chi_{2}(\bmod q)} \sum_{\substack{1 \leq a \leq q \\(a, q)=1}} G\left(a, \bar{\chi}_{1}\right)
$$

$\times G\left(a, \bar{\chi}_{2}\right) G\left(a, \tilde{\chi} \chi_{0}\right) e(-a N / q)$

$$
\begin{aligned}
& \times \sum_{\left|\gamma_{1}\right| \leq T}^{\prime} \sum_{\left|\gamma_{2}\right| \leq T}^{\prime} \int_{\substack{|\eta| \leq \delta(N, q) \\
+}} J\left(\varrho_{1}, \eta\right) J\left(\varrho_{2}, \eta\right) J(\widetilde{\beta}, \eta) e(-N \eta) d \eta \\
& +3 \widetilde{E} \sum_{\substack{1 \leq q \leq P \\
\widetilde{r} \mid q}} \frac{\mu(q)}{\varphi(q)^{3}} \sum_{\substack{1 \leq a \leq q \\
(a, q)=1}} G\left(a, \widetilde{\chi} \chi_{0}\right)^{2} e(-a N / q) \\
& \times \int_{|\eta| \leq \delta(N, q)} J(\eta) J(\widetilde{\beta}, \eta)^{2} e(-N \eta) d \eta \\
& -3 \widetilde{E} \sum_{\substack{1 \leq q \leq P \\
\widetilde{r} \mid q}} \frac{1}{\varphi(q)^{3}} \sum_{\chi(\bmod q)} \sum_{\substack{1 \leq a \leq q \\
(a, q)=1}} G(a, \bar{\chi}) G\left(a, \widetilde{\chi} \chi_{0}\right)^{2} e(-a N / q)
\end{aligned}
$$




$$
\begin{aligned}
& \times \sum_{|\gamma| \leq T}^{\prime} \int_{|\eta| \leq \delta(N, q)} J(\varrho, \eta) J(\widetilde{\beta}, \eta)^{2} e(-N \eta) d \eta \\
& -\widetilde{E} \sum_{\substack{1 \leq q \leq P \\
\widetilde{r} \mid q}} \frac{1}{\varphi(q)^{3}} \sum_{\substack{1 \leq a \leq q \\
(a, q)=1}} G\left(a, \widetilde{\chi} \chi_{0}\right)^{3} e(-a N / q) \\
& \times \int_{|\eta| \leq \delta(N, q)} J(\widetilde{\beta}, \eta)^{3} e(-N \eta) d \eta+R_{13} \\
= & \sum_{1 \leq j \leq 10} I_{1 j}(N)+R_{13},
\end{aligned}
$$

where $\widetilde{E}=1$ if the $\widetilde{\beta}$ in (3.20) exists, and $\widetilde{E}=0$ if it does not exist.

4. A lower bound for $I_{1}(N)$. In this section we shall give an explicit lower bound for $I_{1}(N)$ defined as in (3.11). To this end, we first present two auxiliary lemmas.

Lemma 4.1. For any complex numbers $\varrho_{j}$ with $0<\operatorname{Re} \varrho_{j} \leq 1$ for $1 \leq$ $j \leq 3$, we have

$$
\int_{-\infty}^{\infty} e(-N \eta) \prod_{j=1}^{3} J\left(\varrho_{j}, \eta\right) d \eta=N^{2} \int_{\mathcal{D}} \prod_{j=1}^{3}\left(N x_{j}\right)^{\varrho_{j}-1} d x_{1} d x_{2},
$$

where $x_{3}=1-x_{1}-x_{2}$ and $\mathcal{D}:=\left\{\left(x_{1}, x_{2}\right): 0.001 \leq x_{j} \leq 1\right.$ for $\left.1 \leq j \leq 3\right\}$. 4.7].

Proof. It can be proved by precisely the same way as in [LT, Lemma

Lemma 4.2. Let $J(\eta)$ and $J(\varrho, \eta)$ be defined as in (3.22). Then

$$
|J(\eta)| \leq \min \left\{0.999 N,(\pi|\eta|)^{-1}\right\}
$$

and if $\varrho=\beta+i \gamma$,

$$
\begin{aligned}
& |J(\varrho, \eta)| \\
& \leq \begin{cases}\min \left\{\left(1-0.001^{\beta}\right) \beta^{-1} N^{\beta},\right. & \left.(0.001 N)^{\beta-1}(\pi|\eta|)^{-1}\right\} \quad \text { if } \gamma=0, \\
5 N^{\beta}|\gamma|^{-1} & \text { if }|\eta| \leq|\gamma| /(10 \pi N), \\
16(0.001)^{\beta-1} N^{\beta}|\gamma|^{-1 / 2} & \text { if }|\gamma| /(10 \pi N) \leq|\eta| \leq|\gamma| /(0.001 \pi N), \\
(4 / \pi)(0.001 N)^{\beta-1}|\eta|^{-1} & \text { if }|\eta| \geq|\gamma| /(0.001 \pi N) .\end{cases}
\end{aligned}
$$

Proof. (4.1) and the first inequality in (4.2) are either trivial estimates or consequences of integration by parts. The other three inequalities in (4.2) can be proved in exactly the same way as in [LT, Lemma 3.2], with the help of [T, Lemmas 4.3 and 4.5]. 
Now we give a lower bound for $I_{1}(N)$. Note that by the definition of $\mathcal{D}$ in Lemma 4.1 we have

$$
|\mathcal{D}|:=\int_{\mathcal{D}} d x_{1} d x_{2}=\int_{0.001}^{0.998} d x_{1} \int_{0.001}^{0.999-x_{1}} d x_{2}=\frac{0.997^{2}}{2} .
$$

We first estimate $I_{11}(N)$ defined as in (3.29). Firstly we extend the range of integration with respect to $\eta$ in it to $(-\infty, \infty)$. By (3.3) and (4.1), the total error caused by this extension has absolute value at most $\leq \pi^{-3} \delta(N, q)^{-2}=$ $\pi^{-3}(10 \pi q N /(3.36 P))^{2}$. Thus its contribution to $I_{11}(N)$ has absolute value at most

$$
\begin{aligned}
& \leq \sum_{1 \leq q \leq P} \frac{|\mu(q)|}{\varphi(q)^{2}} \pi^{-3}(10 \pi q N /(3.36 P))^{2} \\
& \leq 2.82 N^{2} P^{-2} \sum_{1 \leq q \leq P}|\mu(q)| q^{2} \varphi(q)^{-2} .
\end{aligned}
$$

Now for any real $x \geq 3$ put $\nu(x):=e^{\gamma} \log \log x+2.50637 / \log \log x$ where $\gamma=0.5772 \ldots$ is the Euler constant. Note that $\nu(x)$ is increasing for $x \geq 27$, and by $[\mathrm{RS} 2,(3.42)]$, we have, for any integer $q \geq 3$,

$$
q / \varphi(q) \leq \nu(q) .
$$

Again, Mathematica yields

$$
\sum_{1 \leq q \leq 1000}|\mu(q)| q^{2} \varphi(q)^{-2} \leq 1961
$$

Thus (4.4) is

$$
\leq 2.82 N^{2} P^{-2}\left(1961+(P-1000) \nu(P)^{2}\right) \leq 2.82 N^{2} P^{-1} \nu(P)^{2} .
$$

If we put

$$
A(q):=\frac{\mu(q)}{\varphi(q)^{3}} \sum_{\substack{1 \leq a \leq q \\(a, q)=1}} e\left(-\frac{a}{q} N\right),
$$

then $I_{11}(N)$ can be rewritten as

$$
I_{11}(N)=\sum_{1 \leq q \leq P} A(q) \int_{-\infty}^{\infty} J(\eta)^{3} e(-N \eta) d \eta+R_{14},
$$

where $\left|R_{14}\right| \leq 2.82 N^{2} P^{-1} \nu(P)^{2}$. Again, by (4.5) we have $\left|\sum_{q>P} A(q)\right| \leq$ $\sum_{q>P} q^{-2} \nu(q)^{2} \leq 1.5 \cdot 10^{-9}$. Thus by Lemma 4.1 with $\varrho_{j}=1$ for $1 \leq j \leq 3$ we may write (4.6) further as

$$
I_{11}(N)=N^{2}|\mathcal{D}| \sum_{q=1}^{\infty} A(q)+1.5 \cdot 10^{-9} \theta N^{2}|\mathcal{D}|+R_{14} .
$$


Note that

$$
\sum_{q=1}^{\infty} A(q)=\prod_{p \mid N}\left(1-(p-1)^{-2}\right) \prod_{p \nmid N}\left(1+(p-1)^{-3}\right),
$$

[D, p. 149], and that, for odd $N$,

$$
\prod_{p \mid N}\left(1-(p-1)^{-2}\right) \geq \prod_{p \geq 3}\left(1-(p-1)^{-2}\right) \geq 0.6601
$$

[HR, p. 128, line -3$]$. Thus by (4.7) and (4.3) we get

(4.8) $\quad I_{11}(N)$

$\geq\left(0.6601 \cdot 0.997^{2}-1.5 \cdot 10^{-9}\left(0.997^{2} / 2\right)-2.82 \cdot 3100^{-3} \nu\left(3100^{3}\right)^{2}\right) N^{2}$ $\geq 0.656145 N^{2}$.

Now we consider two cases according as the $\widetilde{\beta}$ in (3.20) exists or not to estimate $I_{1 j}(N)$ for $2 \leq j \leq 10$.

CASE (I): $\widetilde{\beta}$ does not exist. Firstly consider the estimate of $I_{12}(N)$. By $I_{12}(N)$ in (3.29) and using the well-known bound for $G(a, \chi)$ defined as in (3.14), i.e.,

$$
|G(a, \bar{\chi})| \leq q^{* 1 / 2}
$$

for any $\chi(\bmod q)$ induced by primitive $\chi^{*}\left(\bmod q^{*}\right)$, we get

$$
\begin{aligned}
\left|I_{12}(N)\right| \leq & 3 \sum_{1 \leq q \leq P} \frac{|\mu(q)| q^{1 / 2}}{\varphi(q)^{2}} \\
& \times \sum_{\chi(\bmod q)} \sum_{|\gamma| \leq T}^{\prime}\left|\int_{|\eta| \leq \delta(N, q)} J(\eta)^{2} J(\varrho, \eta) e(-N \eta) d \eta\right| .
\end{aligned}
$$

By Hölder's inequality, the integral in (4.10) has absolute value at most

$$
\left\{\int_{|\eta| \leq \delta(N, q)}|J(\eta)|^{3} d \eta\right\}^{2 / 3}\left\{\int_{|\eta| \leq \delta(N, q)}|J(\varrho, \eta)|^{3} d \eta\right\}^{1 / 3} .
$$

By (4.1), the first integral in (4.11) is, if $1 /(\pi N) \leq \delta(N, q)$,

$$
\begin{aligned}
& \leq \int_{|\eta| \leq \delta(N, q)} \min \left\{0.999 N,(\pi|\eta|)^{-1}\right\}^{3} d \eta \\
& \leq \int_{|\eta| \leq 1 /(\pi N)}(0.999 N)^{3} d \eta+\int_{1 /(\pi N) \leq|\eta| \leq \delta(N, q)}(\pi|\eta|)^{-3} d \eta \\
& \leq(2.994006 / \pi) N^{2} .
\end{aligned}
$$


Note that this bound clearly holds if $1 /(\pi N)>\delta(N, q)$. Substituting this into (4.11), and then into (4.10), we get

$$
\begin{aligned}
\left|I_{12}(N)\right| \leq & 3(2.994006 / \pi)^{2 / 3} N^{4 / 3} \sum_{1 \leq q \leq P} \frac{|\mu(q)| q^{1 / 2}}{\varphi(q)^{2}} \\
& \times \sum_{\chi(\bmod q)} \sum_{|\gamma| \leq T}^{\prime}\left\{\int_{|\eta| \leq \delta(N, q)}|J(\varrho, \eta)|^{3} d \eta\right\}^{1 / 3} .
\end{aligned}
$$

Now we rewrite the last sum over $\gamma$ in (4.13) as

$$
\left\{\sum_{|\gamma| \leq \omega}^{\prime}+\sum_{\omega<|\gamma| \leq T}^{\prime}\right\}\left\{\int_{|\eta| \leq \delta(N, q)}|J(\varrho, \eta)|^{3} d \eta\right\}^{1 / 3} .
$$

For the second sum in (4.14), in view of (3.3) and (2.1), we have $|\eta| \leq$ $\delta(N, q) \leq|\gamma| /(10 \pi N)$. Thus by the second inequality for $J(\varrho, \eta)$ in (4.2), this sum is

$$
\leq 5(3.36 /(5 \pi))^{1 / 3} \mathcal{L} q^{-1 / 3} N^{2 / 3} \sum_{\omega<|\gamma| \leq T}^{\prime} N^{\beta-1}|\gamma|^{-1} .
$$

Again by the first bound for $J(\varrho, \eta)$ in (4.2), the first sum in (4.14) is

$$
\leq(3.36 /(5 \pi))^{1 / 3} \mathcal{L} q^{-1 / 3} N^{2 / 3} \sum_{|\gamma| \leq \omega}^{\prime}\left(1-0.001^{\beta}\right) \beta^{-1} N^{\beta-1} .
$$

Substituting (4.15) and (4.16) into (4.14), and then into (4.13) we get

$$
\begin{aligned}
& \left|I_{12}(N)\right| \leq 3(3.36 /(5 \pi))^{1 / 3}(2.994006 / \pi)^{2 / 3} N^{2} \mathcal{L} \sum_{1 \leq q \leq P} \frac{|\mu(q)| q^{1 / 2}}{\varphi(q)^{2} q^{1 / 3}} \\
& \quad \times\left\{\sum_{\chi(\bmod q)} \sum_{|\gamma| \leq \omega}^{\prime}\left(1-0.001^{\beta}\right) \beta^{-1} N^{\beta-1}+5 \sum_{\chi(\bmod q)} \sum_{\omega<|\gamma| \leq T}^{\prime} N^{\beta-1}|\gamma|^{-1}\right\} .
\end{aligned}
$$

Using Lemmas 2.1 and 2.2 to estimate the first and the second double sums in the last curly brackets respectively we get, for $\mathcal{L} \geq 3100$,

$$
\begin{aligned}
& \left|I_{12}(N)\right| \leq 3(3.36 /(5 \pi))^{1 / 3}(2.994006 / \pi)^{2 / 3} N^{2} \\
& \quad \times\left\{0.0194 \sum_{1 \leq q \leq P} \frac{|\mu(q)| q^{1 / 6}}{\varphi(q)^{2}}+5 \cdot 0.0126 \mathcal{L}^{-3} \sum_{1 \leq q \leq P} \frac{|\mu(q)| q^{1+1 / 6}}{\varphi(q)^{2}}\right\} .
\end{aligned}
$$

Now we need to estimate the two sums over $q$ in the curly brackets in (4.17). Applying Mathematica, we get

$$
\sum_{1 \leq q \leq 10^{5}} \frac{|\mu(q)| q^{1 / 6}}{\varphi(q)^{2}} \leq 3.2842, \quad \sum_{1 \leq q \leq 10^{5}} \frac{|\mu(q)| q^{1+1 / 6}}{\varphi(q)^{2}} \leq 69.9802
$$


By (4.5) we get

$$
\sum_{10^{5}<q \leq P} \frac{q^{1 / 6}}{\varphi(q)^{2}} \leq\left\{\int_{10^{5}}^{10^{10}}+\int_{10^{10}}^{P}\right\} x^{1 / 6-2} \nu(x)^{2} d x .
$$

By Mathematica, the first integral on the right hand side of (4.19) is $\leq$ 0.0025 . When $x \geq 10^{10}$, we have

$$
\nu(x) \leq x^{0.080521}
$$

hence the second integral on the right hand side of (4.19) is

$$
\leq \int_{10^{10}}^{\infty} x^{1 / 6-2+2 \cdot 0.080521} d x \leq 2.82 \cdot 10^{-7}
$$

Thus $(4.19)$ is $\leq 0.0025+2.82 \cdot 10^{-7}$, and consequently by the first inequality in (4.18) we get

$$
\sum_{1 \leq q \leq P} \frac{|\mu(q)| q^{1 / 6}}{\varphi(q)^{2}} \leq 3.2842+0.0025+2.82 \cdot 10^{-7} \leq 3.2868 .
$$

Similarly to (4.19), by (4.5) we get

$$
\sum_{10^{5}<q \leq P} \frac{q^{1+1 / 6}}{\varphi(q)^{2}} \leq\left\{\int_{10^{5}}^{10^{10}}+\int_{10^{10}}^{P}\right\} x^{1 / 6-1} \nu(x)^{2} d x .
$$

By Mathematica, the first integral on the right hand side of (4.22) is $\leq 8794$. The second integral on the right hand side of (4.22) can be estimated as $\leq \nu(P)^{2} \int_{10^{10}}^{P} x^{-5 / 6} d x \leq 6 \nu(P)^{2}\left(P^{1 / 6}-10^{10 / 6}\right)$. This together with $(4.22)$ and the second inequality in (4.18) ensures that

$$
\sum_{1 \leq q \leq P} \frac{|\mu(q)| q^{1+1 / 6}}{\varphi(q)^{2}} \leq 8864+6 \nu(P)^{2}\left(P^{1 / 6}-10^{5 / 3}\right) .
$$

Substituting this and the bounds in (4.21) into (4.17) we get, for $\mathcal{L} \geq 3100$,

$$
\begin{aligned}
\left|I_{12}(N)\right| \leq & 3(3.36 /(5 \pi))^{1 / 3}(2.994006 / \pi)^{2 / 3} N^{2} \\
& \times(0.0194 \cdot 3.2868 \\
& \left.+5 \cdot 0.0126 \mathcal{L}^{-3}\left(8864+6 \nu(P)^{2}\left(P^{1 / 6}-10^{5 / 3}\right)\right)\right) \\
\leq & 0.1108 N^{2} .
\end{aligned}
$$

For the estimates of $I_{13}(N)$ and $I_{14}(N)$, we can proceed in exactly the same way as for $I_{12}(N)$. We have, for $\mathcal{L} \geq 3100$,

$$
\left|I_{13}(N)\right| \leq 0.0016 N^{2} \text { and }\left|I_{14}(N)\right| \leq 0.00002 N^{2} \text {. }
$$

Recall that we are considering the case that $\widetilde{\beta}$ does not exist, so $\widetilde{E}=0$, and hence for $5 \leq j \leq 10$, we have $I_{1 j}(N)=0$. Now by $(3.29)$, (3.28), (4.8), 
(4.23) and (4.24) we can conclude that if $\mathcal{L} \geq 3100$ and if $\widetilde{\beta}$ does not exist, then

$$
\begin{aligned}
I_{1}(N) & \geq(0.656145-0.1108-0.0016-0.00002) N^{2}-3 N^{2} \mathcal{L}^{-9} \\
& \geq 0.5437 N^{2} .
\end{aligned}
$$

CASE (II): $\widetilde{\beta}$ does indeed exist; so $\widetilde{E}=1$. The estimates for $I_{12}(N)$, $I_{13}(N)$ and $I_{14}(N)$ are very similar to those in Case (I): the only difference is that we now may use the second inequality for $\sum_{1}$ in Lemma 2.1 instead of the first one. So with the constant 0.0194 in the estimates of $I_{12}(N)$, $I_{13}(N)$ and $I_{14}(N)$ replaced by the constant $8.2 \cdot 10^{-10}$, we get

$$
\begin{aligned}
& \left|I_{12}(N)\right| \leq 5 \cdot 10^{-8} N^{2}, \quad\left|I_{13}(N)\right| \leq 2 \cdot 10^{-8} N^{2}, \\
& \left|I_{14}(N)\right| \leq 2 \cdot 10^{-8} N^{2} .
\end{aligned}
$$

Now we estimate $I_{15}(N)$. By (3.29) and (4.9), and then using Hölder's inequality we get

$$
\begin{aligned}
\left|I_{15}(N)\right| \leq & 3 \sum_{\substack{1 \leq q \leq P \\
\widetilde{r} \mid q}} \frac{|\mu(q)| \widetilde{r}^{1 / 2}}{\varphi(q)^{2}}\left(\int_{|\eta| \leq \delta(N, q)}|J(\eta)|^{3} d \eta\right)^{2 / 3} \\
& \times\left(\int_{\substack{|\eta| \leq \delta(N, q)\\
}}|J(\widetilde{\beta}, \eta)|^{3} d \eta\right)^{1 / 3} .
\end{aligned}
$$

Note that by (3.20) and $\mathcal{L} \geq 3100$ we have $\widetilde{\beta} \geq 0.9957$. Hence by the first inequality in (4.2), the last integral in (4.27) can be estimated as

$$
\begin{aligned}
& \leq \int_{|\eta| \leq \delta(N, q)} \min \left\{\left(1-0.001^{\widetilde{\beta}}\right) N^{\widetilde{\beta}} \widetilde{\beta}^{-1},(0.001 N)^{\widetilde{\beta}-1}(\pi|\eta|)^{-1}\right\}^{3} d \eta \\
& \leq 3 \cdot 1.0302 \cdot 1.0033^{2} \pi^{-1} N^{3 \widetilde{\beta}-1} .
\end{aligned}
$$

Substituting (4.12) and (4.28) into (4.27) we get, for $\mathcal{L} \geq 3100$,

$$
\left|I_{15}(N)\right| \leq 2.8959 N^{2} N^{\widetilde{\beta}+1} \sum_{\substack{1 \leq q \leq P \\ \widetilde{r} \mid q}} \frac{|\mu(q)| \widetilde{r}^{1 / 2}}{\varphi(q)^{2}} .
$$

Note that $\varphi(m n) \geq \varphi(m) \varphi(n)$. Hence the last sum over $q$ in (4.29) is

$$
\leq \sum_{1 \leq q \leq P / \widetilde{r}} \frac{|\mu(q)| \cdot|\mu(\widetilde{r})| \widetilde{r}^{1 / 2}}{\varphi(\widetilde{r})^{2} \varphi(q)^{2}}=\frac{|\mu(\widetilde{r})| \widetilde{r}^{1 / 2}}{\varphi(\widetilde{r})^{2}} \sum_{1 \leq q \leq P / \widetilde{r}} \frac{|\mu(q)|}{\varphi(q)^{2}} .
$$

By Mathematica and (4.20), similarly to (4.18) and (4.21), the last sum over $q$ in $(4.30)$ is $\leq 2.8265+0.00031+4.9 \cdot 10^{-9} \leq 2.82682$. Hence by $(4.30)$ and 
[LW, Theorem 3] we may rewrite (4.29) further as, for $\mathcal{L} \geq 3100$,

$$
\left|I_{15}(N)\right| \leq 2.8959 \cdot 2.82682 N^{2}\left\{\frac{|\mu(\widetilde{r})| \widetilde{r}^{1 / 2}}{\varphi(\widetilde{r})^{2}} N^{(-\pi / 0.4923) /\left(\widetilde{r}^{1 / 2} \log ^{2} \widetilde{r}\right)}\right\} .
$$

By (4.5), the expression in the last curly brackets is

$$
\leq \widetilde{r}^{-1.5}\left(e^{\gamma} \log \log \widetilde{r}+2.50637 / \log \log \widetilde{r}\right)^{2} e^{-3100 \pi /\left(0.4923 \widetilde{r}^{1 / 2} \log ^{2} \widetilde{r}\right)},
$$

and so in view of $\widetilde{r} \geq 987$, it is, by Mathematica, $\leq 2.5636 \cdot 10^{-6}$. We then infer that, for $\mathcal{L} \geq 3100$,

$$
\left|I_{15}(N)\right| \leq 2.8959 \cdot 2.82682 \cdot 2.5636 \cdot 10^{-6} N^{2} \leq 2.1 \cdot 10^{-5} N^{2} .
$$

For the estimates of $I_{16}(N), I_{17}(N)$ and $I_{19}(N)$, we may use similar arguments as for $I_{12}(N)$ and $I_{15}(N)$. We have, for $\mathcal{L} \geq 3100$,

$$
\begin{aligned}
& \left|I_{16}(N)\right| \leq 4 \cdot 10^{-8} N^{2}, \quad\left|I_{17}(N)\right| \leq 0.00318 N^{2}, \\
& \left|I_{19}(N)\right| \leq 0.0001 N^{2} .
\end{aligned}
$$

For $I_{18}(N)$, by $(3.29),(4.9)$ and Hölder's inequality, and then by (4.12) and (4.28) we get

$$
\left|I_{18}(N)\right| \leq 3\left(2.994006 \pi^{-1}\right)^{1 / 3}\left(3 \cdot 1.0302 \cdot 1.0033^{2} \pi^{-1}\right)^{2 / 3} N^{2 \widetilde{\beta}} \sum_{\substack{1 \leq q \leq P \\ \widetilde{r} \mid q}} \frac{|\mu(q)| \widetilde{r}}{\varphi(q)^{2}} .
$$

This together with (4.5), [LW, Theorem 3] and Mathematica yields

$$
\begin{aligned}
\left|I_{18}(N)\right| & \leq 8.2914 N^{2}\left\{\widetilde{r}^{-1} \nu(\widetilde{r})^{2} e^{-2 \pi \mathcal{L} /\left(0.4923 \widetilde{r}^{1 / 2} \log ^{2} \widetilde{r}\right)}\right\} \\
& \leq 8.2914 \cdot 0.00013 N^{2} \leq 0.00108 N^{2} .
\end{aligned}
$$

For $I_{1,10}(N)$, by $(3.29),(4.9)$ and (4.28), and then using Mathematica, [LW, Theorem 3] and (4.5), we get

$$
\begin{aligned}
\left|I_{1,10}(N)\right| & \leq 3 \cdot 1.0302 \cdot 1.0033^{2} \pi^{-1} \widetilde{r}^{1.5} \varphi(\widetilde{r})^{-2} N^{3 \widetilde{\beta}-1} \sum_{1 \leq q \leq P} \frac{1}{\varphi(q)^{2}} \\
& \leq 3 \cdot 1.0302 \cdot 1.0033^{2} \cdot 3.39102 \pi^{-1} N^{2} \widetilde{r}^{1.5} \varphi(\widetilde{r})^{-2} N^{3 \widetilde{\beta}-3} \\
& \leq 3.35804 N^{2}\left\{\widetilde{r}^{-1 / 2} \nu(\widetilde{r})^{2} e^{-3 \pi \mathcal{L} /\left(0.4923 \widetilde{r}^{1 / 2} \log ^{2} \widetilde{r}\right)}\right\} \\
& \leq 3.35804 \cdot 0.028 N^{2} \leq 0.09403 N^{2} .
\end{aligned}
$$

This together with (3.29), (3.28), (4.8), (4.26) and (4.31) to (4.33) ensures that if $\mathcal{L} \geq 3100$ and if $\widetilde{\beta}$ exists then

$$
\begin{aligned}
I_{1}(N) \geq & \left(0.656145-5 \cdot 10^{-8}-2 \cdot 10^{-8}-2 \cdot 10^{-8}\right. \\
& -2.1 \cdot 10^{-5}-4 \cdot 10^{-8}-0.00318-0.00108 \\
& -0.0001-0.09403) N^{2}-3 N^{2} \mathcal{L}^{-9} \\
\geq & 0.5577 N^{2} .
\end{aligned}
$$


From (4.25) and (4.34) we can conclude the following

Lemma 4.3. Let $I_{1}(N)$ be defined as in (3.11). Then for $N \geq \exp (3100)$ we have

$$
I_{1}(N) \geq 0.5437 N^{2}
$$

5. Trigonometric sums over primes (I). In this section we shall give explicit upper bound estimates for the trigonometric sums $S(\alpha)$ defined by (3.8) when the $q$ in (3.2) is small. More precisely, we shall bound $S(\alpha)$ when $\alpha$ is in $\mathcal{M}_{2}$ and $\mathcal{M}_{3}$, which are defined by (3.5) and (3.6) respectively.

Lemma 5.1. Let $S(\alpha)$ and $\mathcal{M}_{2}$ be defined as in (3.8) and (3.5). Then for $\alpha \in \mathcal{M}_{2}$ and $\mathcal{L} \geq 3100$ we have

$$
|S(\alpha)| \leq 0.4012 N \mathcal{L}^{-1} .
$$

Proof. By (3.19) with $T=\mathcal{L}^{15}$ (in (2.1)), (3.21) and (4.9) we have

$$
\begin{aligned}
|S(\alpha)| \leq & \frac{|\mu(q)|}{\varphi(q)}|J(\eta)|+\frac{\delta(q)}{\varphi(q)} \widetilde{r}^{1 / 2}|J(\widetilde{\beta}, \eta)| \\
& +\frac{q^{1 / 2}}{\varphi(q)} \sum_{\chi(\bmod q)} \sum_{|\gamma| \leq T}^{\prime}|J(\varrho, \eta)| \\
& +(1.3818+4.3367 N|\eta|) N \mathcal{L}^{-13} q^{1 / 2}+(\log 2)^{-1} \mathcal{L}^{2},
\end{aligned}
$$

where $\delta(q)=1$ if the $\widetilde{\beta}$ in (3.20) exists with $\widetilde{r} \mid q$, and $\delta(q)=0$ otherwise. Note that by (3.2), (3.3) and (3.5) we have, for $\alpha=a / q+\eta \in \mathcal{M}_{2}$,

$$
1 \leq q \leq P \text { and } 3.36 P /(10 \pi q N) \leq|\eta| \leq 1 /(q Q) .
$$

From this, (4.1), (4.5), $\widetilde{r} \leq P$ and the first inequality in (4.2) we see that the sum of the first two terms on the right hand side of (5.1) is $\leq(10 N /(3.36 P)) \nu(P)+\delta(q)\left(10 N /\left(3.36 P^{1 / 2}\right)\right) \nu(P)$. In view of $Q=N \mathcal{L}^{-7}$ (in (3.1)), the sum of the last two terms on the right hand side of (5.1) is $\leq 4.3368 N \mathcal{L}^{-6}$. Further, write the $\operatorname{sum} \sum_{|\gamma| \leq T}^{\prime}$ in (5.1) as

$$
\sum_{|\gamma| \leq \omega}^{\prime}+\sum_{\omega<|\gamma| \leq 10 \pi \mathcal{L}^{7} q^{-1}}^{\prime}+\sum_{10 \pi \mathcal{L}^{7} q^{-1}<|\gamma| \leq T}^{\prime} .
$$

Using (4.2) to estimate $|J(\varrho, \eta)|$ and using the bounds $q^{1 / 2} \varphi(q)^{-1} \leq \sqrt{2}$ and $q^{1.5} \varphi(q)^{-1} \leq P^{0.5} \nu(P)$ for any integer $q \geq 1$, we get, by Lemmas 2.1 and 2.2 ,

$$
\begin{aligned}
|S(\alpha)| \leq & (10 N /(3.36 P)) \nu(P)+\left(10 N /\left(3.36 P^{1 / 2}\right)\right) \nu(P) \\
& +4.3368 N \mathcal{L}^{-6}+8.2 \sqrt{2} \cdot 10^{-10} N \mathcal{L}^{-1} \\
& +5 \cdot 0.0126 \nu\left(\mathcal{L}^{3}\right) N \mathcal{L}^{-2.5}+q^{1 / 2} \varphi(q)^{-1} \sum_{3} \\
\leq & 0.3452 N \mathcal{L}^{-1}+q^{1 / 2} \varphi(q)^{-1} \sum_{3}
\end{aligned}
$$


where

$$
\sum_{3}:=\sum_{\chi(\bmod q)} \sum_{\omega \leq|\gamma| \leq 10 \pi \mathcal{L}^{7} q^{-1}}^{\prime}|J(\varrho, \eta)| .
$$

When $|\gamma| \geq 1$, it is easy to verify that the third inequality in (4.2) gives the weakest estimate for $|J(\varrho, \eta)|$ among the last three estimates in (4.2). So it can be applied in any case. And thus we can use the third inequality in (4.2) to obtain

$$
\begin{aligned}
\sum_{3} \leq & 16 N\left\{(0.001 N)^{-1 / 2} \sum_{\chi(\bmod q)} \sum_{\omega \leq|\gamma| \leq 10 \pi \mathcal{L}^{7} q^{-1}}^{\prime}|\gamma|^{-1 / 2}\right. \\
& +\left(\int_{1 / 2}^{59 / 60}+\int_{59 / 60}^{1}\right)(0.001 N)^{\alpha-1}(\log 0.001 N) \\
& \left.\times \sum_{\chi(\bmod q)} \sum_{\substack{\omega \leq|\gamma| \leq 10 \pi \mathcal{L}^{7} q^{-1} \\
\beta \geq \alpha}}^{\prime}|\gamma|^{-1 / 2} d \alpha\right\}
\end{aligned}
$$

Similarly to $(2.19)$, for any $\alpha \in[1 / 2,1)$ we have

$$
\begin{aligned}
\sum_{\chi(\bmod q)} \sum_{\substack{\omega \leq|\gamma| \leq 10 \pi \mathcal{L}^{7} q^{-1} \\
\beta \geq \alpha}}|\gamma|^{-1 / 2} & =\int_{\omega}^{10 \pi \mathcal{L}^{7} q^{-1}} y^{-1 / 2} d N(\alpha, q, y) \\
\leq & \left(10 \pi \mathcal{L}^{7} q^{-1}\right)^{-1 / 2} N\left(\alpha, q, 10 \pi \mathcal{L}^{7} q^{-1}\right) \\
& +\frac{1}{2} \int_{\omega}^{10 \pi \mathcal{L}^{7} q^{-1}} y^{-3 / 2} N(\alpha, q, y) d y
\end{aligned}
$$

If we use the bound in (2.4) with $q=P$ (so $\omega=3.36$ in $(2.1)$ ), (2.4) can be estimated further as $\leq 2(10 / \pi)^{1 / 2} \mathcal{L}^{3.5} q^{-0.5} \varphi(q) \log \left(10 \pi \mathcal{L}^{10}\right)$. Thus the sum of the first term and the first integral on the right hand side of (5.3) is

$$
\begin{aligned}
\leq & \left\{(0.001 N)^{-1 / 2}+\int_{1 / 2}^{59 / 60}(0.001 N)^{\alpha-1} \log (0.001 N) d \alpha\right\} \\
& \times 2(10 / \pi)^{1 / 2} \mathcal{L}^{3.5} q^{-0.5} \varphi(q) \log \left(10 \pi \mathcal{L}^{10}\right) \\
\leq & 0.000065 q^{-0.5} \varphi(q) \mathcal{L}^{-1} .
\end{aligned}
$$

Note that by [LW, Theorem 7] we have, for $59 / 60 \leq \alpha<1$,

$$
\begin{aligned}
N\left(\alpha, q, 10 \pi \mathcal{L}^{7} q^{-1}\right) \leq & \left(254231 / \log \left(10 \pi \mathcal{L}^{7}\right)+17102\right) \\
& \times\left(\left(10 \pi \mathcal{L}^{7}\right)^{4} q^{-1}\right)^{1 / 60} \log ^{6}\left(10 \pi \mathcal{L}^{7}\right)+16541 \log ^{6}\left(10 \pi \mathcal{L}^{7}\right) .
\end{aligned}
$$

By this inequality and the bound $7000 \cdot 2$ in [LW, Table 5], the contribution to the last integral on the right hand side of (5.3) from the first term on the 
right hand side of (5.4) is

$$
\begin{aligned}
\leq & \left(10 \pi \mathcal{L}^{7}\right)^{-1 / 2+1 / 15} q^{1 / 2-1 / 60}(0.001 N)^{-0.478 / \log \left(10 \pi \mathcal{L}^{7}\right)} \\
& \times\left(254231 / \log \left(10 \pi \mathcal{L}^{7}\right)+17102\right) \log ^{6}\left(10 \pi \mathcal{L}^{7}\right) \\
& +16541\left(10 \pi \mathcal{L}^{7}\right)^{-1 / 2} q^{1 / 2}(0.001 N)^{-0.478 / \log \left(10 \pi \mathcal{L}^{7}\right)} \log ^{6}\left(10 \pi \mathcal{L}^{7}\right) \\
& +14000(10 \pi)^{-1 / 2} \mathcal{L}^{-3.5} q^{0.5}(0.001 N)^{-0.10367089 / \log \left(10 \pi \mathcal{L}^{7}\right)},
\end{aligned}
$$

where 0.10367089 comes from $1 / c_{1}$ (in [LW, Lemma 2.1]). On noting $q^{1-1 / 60} \varphi(q)^{-1} \leq 4.4772$ and $q \varphi(q)^{-1} \leq \nu(P)$ for any $1 \leq q \leq P$, (5.6) is

$$
\leq\left(0.02198+0.00046+4.5 \cdot 10^{-6}\right) N \mathcal{L}^{-1} \leq 0.022445 N \mathcal{L}^{-1}
$$

This together with (5.2) to (5.5) yields

$$
\begin{aligned}
|S(\alpha)| \leq & (0.3452+16 \cdot 0.000065+0.022445) N \mathcal{L}^{-1} \\
& +8 N q^{1 / 2} \varphi(q)^{-1} \int_{\omega}^{10 \pi \mathcal{L}^{7} q^{-1}} y^{-3 / 2} \\
& \times \int_{59 / 60}^{1}(0.001 N)^{\alpha-1}(\log 0.001 N) N(\alpha, q, y) d \alpha d y .
\end{aligned}
$$

By [LW, Lemma 2.1], the innermost integral on the right hand side of (5.7) can be rewritten as

$$
\int_{59 / 60}^{1-1 /\left(c_{1} \log q y\right)}=\int_{59 / 60}^{1-0.478 / \log q y}+\int_{1-0.478 / \log q y}^{1-1 /\left(c_{1} \log q y\right)} .
$$

We first consider the contribution to (5.7) from the first integral on the right hand side of (5.8). Write

$$
M_{1}:=\int_{\omega}^{10 \pi \mathcal{L}^{7} q^{-1}} y^{-3 / 2} \int_{59 / 60}^{1-0.478 / \log q y}(0.001 N)^{\alpha-1}(\log 0.001 N) N(\alpha, q, y) d \alpha d y
$$

We consider two cases according as $\omega \geq \max \left(10^{5} q^{-1}, 10^{4} \log q\right)$ or not. If $\omega \geq \max \left(10^{5} q^{-1}, 10^{4} \log q\right.$ ) (so is $y$ ), then by [LW, Theorem 7 ], the innermost integral in $M_{1}$ is

$$
\begin{aligned}
\leq & \left(\frac{254231}{\log q y}+33643\right)(\log q y)^{6} \frac{\log 0.001 N}{\log \left(0.001 N q^{-3} y^{-4}\right)} \\
& \times\left(0.001 N q^{-3} y^{-4}\right)^{-0.478 / \log q y}
\end{aligned}
$$

thus by (2.1) and since $q \varphi(q)^{-1} \leq \nu(P)$ for $1 \leq q \leq P$, 


$$
\begin{aligned}
8 q^{1 / 2} \varphi(q)^{-1} & M_{1} \\
\leq & \mathcal{L}^{-1}\left\{8 e^{4 \cdot 0.478} \nu\left(\mathcal{L}^{3}\right) \mathcal{L}\right. \\
& \times \int_{\log \left(3.36 \mathcal{L}^{3}\right)}^{\log \left(10 \pi \mathcal{L}^{7}\right)}(254231 / y+33643) \frac{y^{6}(\mathcal{L}+\log 0.001)}{\mathcal{L}-4 y+\log 0.001} \\
& \left.\times e^{-y / 2-0.478(\mathcal{L}+\log 0.001) / y} d y\right\} .
\end{aligned}
$$

By Mathematica, the expression in the last curly brackets, as a function of $\mathcal{L}$, is shown to be decreasing, and with $\mathcal{L}=3100$, it can be estimated as

$$
\leq 0.032281 \text {. }
$$

If $\omega<\max \left(10^{5} q^{-1}, 10^{4} \log q\right)$, we rewrite $M_{1}$ as

$$
M_{1}=\int_{\omega}^{\max \left(10^{5} q^{-1}, 10^{4} \log q\right)}+\int_{\max \left(10^{5} q^{-1}, 10^{4} \log q\right)}^{10 \pi \mathcal{L}^{7} q^{-1}} .
$$

If we use the bound in (2.4) with $q=P$ to estimate $N(\alpha, q, y)$, the first integral on the right hand side of (5.11) is, by noting $1 \leq q \leq P$,

$$
\begin{aligned}
\leq & q^{-1 / 2} \varphi(q) \mathcal{L}^{-1} \int_{3.36 P}^{10^{4} P \log P} \mathcal{L}\left(\pi^{-1} y^{-1 / 2} \log P y-0.874 y^{-1 / 2}\right. \\
& \left.+6.8423 y^{-3 / 2} P \log P y+15 y^{-3 / 2} P\right) e^{-0.478(\log 0.001 N) / \log y} d y .
\end{aligned}
$$

As $P=\mathcal{L}^{3}$ and $\mathcal{L}=\log N$, by Mathematica, the last integral takes its supremum at $\mathcal{L}=3100$ if $\mathcal{L} \geq 3100$; and for $\mathcal{L}=3100$, it is $\leq 7.6 \cdot 10^{-6}$. So (5.12) is

$$
\leq 7.6 \cdot 10^{-6} q^{-1 / 2} \varphi(q) \mathcal{L}^{-1}
$$

For the second integral on the right hand side of (5.11), we do have $y \geq$ $\max \left(10^{5} q^{-1}, 10^{4} \log q\right)$. So we can use [LW, Theorem 7$]$ to estimate $N(\alpha, q, y)$ completely as in the above case where $\omega \geq \max \left(10^{5} q^{-1}, 10^{4} \log q\right)$. Then we replace the lower integral bound $\max \left(10^{5} q^{-1}, 10^{4} \log q\right)$ in the second integral on the right hand side of $(5.11)$ by $\omega$ since in this case $\omega \leq$ $\max \left(10^{5} q^{-1}, 10^{4} \log q\right)$. In this way, we see that the second integral on the right hand side of (5.11) can be bounded exactly by the bound for $M_{1}$ implied by (5.9). Thus by (5.9) and (5.10), this integral is

$$
\leq(0.032281 / 8) q^{-1 / 2} \varphi(q) \mathcal{L}^{-1} .
$$

Now by (5.11) and (5.13) we get

$$
8 q^{1 / 2} \varphi(q)^{-1} M_{1} \leq\left(0.032281+8 \cdot 7.6 \cdot 10^{-6}\right) \mathcal{L}^{-1} .
$$


This in combination with (5.7) and (5.8) ensures that

$$
\begin{aligned}
|S(\alpha)| & \leq\left(0.3687+0.032281+8 \cdot 7.6 \cdot 10^{-6}\right) N \mathcal{L}^{-1}+M_{2} \\
& \leq 0.4011 N \mathcal{L}^{-1}+M_{2}
\end{aligned}
$$

where

$$
\begin{aligned}
M_{2}:= & 8 N q^{1 / 2} \varphi(q)^{-1} \int_{\omega}^{10 \pi \mathcal{L}^{7} q^{-1}} y^{-3 / 2} \\
& \times \int_{1-0.478 / \log q y}^{1-1 /\left(c_{1} \log q y\right)}(0.001 N)^{\alpha-1}(\log 0.001 N) N(\alpha, q, y) d \alpha d y .
\end{aligned}
$$

Now we rewrite the innermost integral $\int_{1-0.478 / \log q y}^{1-1 /\left(c_{1} \log q y\right)}$ as

$$
\int_{1-0.478 / \log q y}^{1-0.2067 / \log q y}+\int_{1-0.2067 / \log q y}^{1-1 /\left(c_{1} \log q y\right)} .
$$

By (2.1), (4.5) and the bound $7000 \cdot 2$ in [LW, Table 5], the contribution to $M_{2}$ from the first integral in (5.15) is

$$
\begin{aligned}
\leq & 112000 N \mathcal{L}^{-1}\left\{\nu\left(\mathcal{L}^{3}\right) \mathcal{L} \int_{\log \left(3.36 \mathcal{L}^{3}\right)}^{\log \left(10 \pi \mathcal{L}^{7}\right)} \exp (-0.5 y)\right. \\
& \times\left(\exp \left(-\frac{0.2067(\mathcal{L}+\log 0.001)}{y}\right)\right. \\
& \left.\left.-\exp \left(-\frac{0.478(\mathcal{L}+\log 0.001)}{y}\right)\right) d y\right\} \\
\leq & 2.1 \cdot 10^{-5} N \mathcal{L}^{-1}
\end{aligned}
$$

By [LW, Theorem 2], the contribution to $M_{2}$ from the last integral in (5.15) is

$$
\begin{aligned}
& \leq 2 \cdot 8 N q^{1 / 2} \varphi(q)^{-1} \int_{\omega}^{10 \pi \mathcal{L}^{7} q^{-1}} y^{-3 / 2} \\
& \quad \times \int_{1-0.2067 / \log q y}^{1-1 /\left(c_{1} \log q y\right)}(0.001 N)^{\alpha-1}(\log 0.001 N) d \alpha d y \\
& \leq 2.4 \cdot 10^{-5} N \mathcal{L}^{-1} .
\end{aligned}
$$

From (5.14), (5.16) and (5.17) we get

$$
|S(\alpha)| \leq\left(0.4011+2.1 \cdot 10^{-5}+2.4 \cdot 10^{-5}\right) N \mathcal{L}^{-1} \leq 0.4012 N \mathcal{L}^{-1} .
$$

The proof of Lemma 5.1 is complete. 
Lemma 5.2. Let $S(\alpha)$ and $\mathcal{M}_{3}$ be defined as in (3.8) and (3.6). Then for $\alpha \in \mathcal{M}_{3}$ and $\mathcal{L} \geq 3100$ we have

$$
|S(\alpha)| \leq 0.5033 N \mathcal{L}^{-1} .
$$

Proof. Note that for $\alpha \in \mathcal{M}_{3}$ we have

$$
\alpha=a / q+\eta, \quad \mathcal{L}^{3} \leq q \leq \mathcal{L}^{6},|\eta| \leq 1 /(q Q)=\mathcal{L}^{7}(q N)^{-1} .
$$

By (3.18), (3.19) with $T=\mathcal{L}^{15}$ (in (2.1)), (4.5) and (4.9) we have

$$
|S(\alpha)| \leq\left(6.8 \cdot 10^{-7}+9 \cdot 10^{-23}\right) N \mathcal{L}^{-1}+\frac{q^{1 / 2}}{\varphi(q)} \sum_{\chi(\bmod q)} \sum_{\substack{|\gamma| \leq T \\ \beta \geq 1 / 2}}|J(\varrho, \eta)| .
$$

In view of $|\eta| \leq \mathcal{L}^{7} /(q N)$ in (5.18), by (4.2) we have $|J(\varrho, \eta)| \leq 5 N^{\beta}|\gamma|^{-1}$ for $|\gamma| \geq 10 \pi \mathcal{L}^{7} q^{-1}$. For $10^{4} \pi \leq|\gamma| \leq 10 \pi \mathcal{L}^{7} q^{-1}$, the next-to-last inequality in (4.2) gives the worst estimate for $J(\varrho, \eta)$ among the last three estimates in (4.2). So for this case, we can use the next-to-last inequality in (4.2) to obtain $|J(\varrho, \eta)| \leq 16(0.001)^{\beta-1} N^{\beta}|\gamma|^{-1 / 2}$. Also, we have the bound $|J(\varrho, \eta)|$ $\leq\left(1-0.001^{\beta}\right) \beta^{-1} N^{\beta}$ for $|\gamma| \leq 10^{4} \pi$. Thus (5.19) can be rewritten as

$$
\begin{aligned}
|S(\alpha)| \leq & \left(6.8 \cdot 10^{-7}+9 \cdot 10^{-23}\right) N \mathcal{L}^{-1} \\
& +\frac{q^{1 / 2}}{\varphi(q)} \sum_{\chi(\bmod q)} \sum_{\substack{|\gamma| \leq 10^{4} \pi \\
\beta \geq 1 / 2}}\left(1-0.001^{\beta}\right) \beta^{-1} N^{\beta} \\
& +\frac{16 q^{1 / 2}}{\varphi(q)} \sum_{\chi(\bmod q)} \sum_{\substack{10^{4} \pi \leq|\gamma| \leq 10 \pi \mathcal{L}^{7} q^{-1} \\
\beta \geq 1 / 2}}(0.001)^{\beta-1} N^{\beta}|\gamma|^{-1 / 2} \\
& +\frac{5 q^{1 / 2}}{\varphi(q)} \sum_{\chi(\bmod q)} \sum_{\substack{10 \pi \mathcal{L}^{7} q^{-1} \leq|\gamma| \leq T \\
\beta \geq 1 / 2}} N^{\beta}|\gamma|^{-1} \\
= & :\left(6.8 \cdot 10^{-7}+9 \cdot 10^{-23}\right) N \mathcal{L}^{-1}+\sum_{4}+\sum_{5}+\sum_{6} .
\end{aligned}
$$

Now we estimate $\sum_{4}, \sum_{5}$ and $\sum_{6}$. We first estimate $\sum_{4}$. By [LW, Lemma 2.1 with $\left.x=10^{4} \pi q\right]$ we know that for any zero $\varrho=\beta+i \gamma$ with $|\gamma| \leq 10^{4} \pi$ of any $L(s, \chi)$ with $\chi(\bmod q)$, there exists $\beta \leq 1-1 /\left(c_{1} \log 10^{4} \pi q\right)$ except for at most one possible real zero $\widetilde{\beta}_{1}$ corresponding to a real character $\widetilde{\chi}_{1}$ $\left(\bmod \widetilde{r}_{1}\right)$. Thus in view of $1 / 2 \leq \widetilde{\beta}_{1}<1, \mathcal{L}^{3} \leq q \leq \mathcal{L}^{6}$ and $(4.5)$,

$$
\begin{aligned}
\sum_{4} & \leq q^{1 / 2} \varphi(q)^{-1}\left(1-0.001^{\widetilde{\beta}_{1}}\right) \widetilde{\beta}_{1}^{-1} N^{\widetilde{\beta}_{1}}+\sum_{4}^{\prime} \\
& \leq 0.11585 N \mathcal{L}^{-1}+\sum_{4}^{\prime},
\end{aligned}
$$


where the ${ }^{\prime}$ in $\sum^{\prime}$ indicates that the $\widetilde{\beta}_{1}$ is excluded and

$$
\sum_{4}^{\prime}:=q^{1 / 2} \varphi(q)^{-1} N \sum_{\chi(\bmod q)} \sum_{\substack{|\gamma| \leq 10^{4} \pi \\ \beta \geq 1 / 2}}^{\prime}\left(1-0.001^{\beta}\right) \beta^{-1} N^{\beta-1} .
$$

Similarly to $(2.2)$ we have

$$
\begin{aligned}
& \text { 5.22) } \quad \sum_{4}^{\prime} \leq 2\left(1-0.001^{1 / 2}\right) q^{1 / 2} N \varphi(q)^{-1} N^{-1 / 2} N\left(1 / 2, q, 10^{4} \pi\right) \\
& +\frac{q^{1 / 2} N}{\varphi(q)}\left\{\int_{1 / 2}^{59 / 60}+\int_{59 / 60}^{1-0.478 / \log \left(10^{4} \pi q\right)}+\int_{1-0.478 / \log \left(10^{4} \pi q\right)}^{1-0.2067 / \log \left(10^{4} \pi q\right)}+\int_{1-0.2067 / \log \left(10^{4} \pi q\right)}^{1-1 /\left(c_{1} \log \left(10^{4} \pi q\right)\right)}\right\}
\end{aligned}
$$

$\times N\left(\alpha, q, 10^{4} \pi\right) N^{\alpha-1}(\log N)\left(1-0.001^{\alpha}\right) \alpha^{-1} d \alpha$.

Note that the bound in (2.4) is always greater than that in (2.3) if $y=10^{4} \pi$ and $\mathcal{L}^{3} \leq q \leq \mathcal{L}^{6}$. So for any $\alpha \in[1 / 2,1)$, by $(2.4)$ we have $N\left(\alpha, q, 10^{4} \pi\right) \leq$ $\varphi(q)(10006.8423 \log q+76180)$. Thus the sum of the first two terms on the right hand side of $(5.22)$ is

$$
\begin{aligned}
\leq & 2\left(1-0.001^{1 / 2}\right) N q^{1 / 2}(10006.8423 \log q+76180) \\
& \times\left(N^{-1 / 2}+\int_{1 / 2}^{59 / 60} N^{\alpha-1} \log N d \alpha\right) \\
\leq & 0.00365 N \mathcal{L}^{-1} .
\end{aligned}
$$

For the second integral on the right hand side of (5.22), we note that by [LW, Theorem 7],

$$
\begin{aligned}
N\left(\alpha, q, 10^{4} \pi\right) \leq & N\left(\alpha, q, 10^{4} \log q\right) \\
\leq & 16541 \log ^{6}\left(10^{4} \log q\right)+\left(\frac{254231}{\log \left(10^{4} q \log q\right)}+17102\right) \\
& \times\left(q^{3} 10^{16} \log ^{4} q\right)^{1-59 / 60} \log ^{6}\left(10^{4} q \log q\right) ;
\end{aligned}
$$

thus its contribution to the right hand side of (5.22) is

$$
\begin{aligned}
\leq & 1.8771 N q^{-9 / 20} \nu(q)(\log q)^{1 / 15} \\
& \times\left(\frac{254231}{\log \left(10^{4} q \log q\right)}+17102\right) \log ^{6}\left(10^{4} q \log q\right) e^{-0.478 \mathcal{L} / \log \left(10^{4} \pi q\right)} \\
& +1.0159 \cdot 16541 N q^{-1 / 2} \nu(q) \log ^{6}\left(10^{4} \log q\right) \exp \left(-\frac{0.478 \mathcal{L}}{\log \left(10^{4} \pi q\right)}\right) .
\end{aligned}
$$

For $q \in\left[\mathcal{L}^{3}, \mathcal{L}^{6}\right]$, let $q=\mathcal{L}^{x}$ with $3 \leq x \leq 6$. Then this is

$$
\begin{array}{r}
\leq N \mathcal{L}^{-1}\left\{\left(1 . 8 7 7 1 \mathcal { L } ^ { 1 - 9 x / 2 0 } \nu ( \mathcal { L } ^ { x } ) ( x \operatorname { l o g } \mathcal { L } ) ^ { 1 / 1 5 } \left(254231 / \log \left(10^{4} \mathcal{L}^{x} \log \mathcal{L}^{x}\right)\right.\right.\right. \\
+17102) \log ^{6}\left(10^{4} \mathcal{L}^{x} \log \mathcal{L}^{x}\right)
\end{array}
$$




$$
\left.\left.+1.0159 \cdot 16541 \mathcal{L}^{1-x / 2} \nu\left(\mathcal{L}^{x}\right) \log ^{6}\left(10^{4} x \log \mathcal{L}\right)\right) \exp \left(-\frac{0.478 \mathcal{L}}{\log \left(10^{4} \pi \mathcal{L}^{x}\right)}\right)\right\} .
$$

By Mathematica, the "Plot 3D procedure", the expression in the last curly brackets, as a function of $\mathcal{L}$ and $x$, has upper bound 0.24981 for $\mathcal{L} \geq 3100$ and $3 \leq x \leq 6$. So the above is

$$
\leq 0.24981 N \mathcal{L}^{-1} \text {. }
$$

For the third integral on the right hand side of (5.22), we may use the bound $7000 \cdot 2$ in [LW, Table 5] to estimate $N\left(\alpha, q, 10^{4} \pi\right)$. So its contribution to the right hand side of $(5.22)$ is

$$
\leq 14000 \cdot \frac{60\left(1-0.001^{59 / 60}\right)}{59} N q^{-1 / 2} \nu(q) N^{-0.2067 / \log \left(10^{4} \pi q\right)} .
$$

If we let $q=\mathcal{L}^{x}$ with $3 \leq x \leq 6$, this is

$$
\begin{aligned}
& \leq N \mathcal{L}^{-1}\left\{14000 \cdot \frac{60\left(1-0.001^{59 / 60}\right)}{59} \mathcal{L}^{1-x / 2} \nu\left(\mathcal{L}^{x}\right) e^{-\frac{0.2067 \mathcal{L}}{\log \left(10^{4} \pi \mathcal{L}^{x}\right)}}\right\} \\
& \leq 0.00003 N \mathcal{L}^{-1}
\end{aligned}
$$

for $\mathcal{L} \geq 3100$ by Mathematica. By [LW, Theorem 2], the contribution to the right hand side of (5.22) from the last integral in it is, if $q=\mathcal{L}^{x}$,

$$
\begin{aligned}
& \leq N \mathcal{L}^{-1}\left\{\frac{2\left(1-0.001^{59 / 60}\right)}{59 / 60} \mathcal{L}^{1-x / 2} \nu\left(\mathcal{L}^{x}\right) \exp \left(-\frac{0.10367089 \mathcal{L}}{\log \left(10^{4} \pi \mathcal{L}^{x}\right)}\right)\right\} \\
& \leq 0.00003 N \mathcal{L}^{-1},
\end{aligned}
$$

for $\mathcal{L} \geq 3100$ and $3 \leq x \leq 6$. From (5.21) to (5.26) we can summarize that, for $\mathcal{L} \geq 3100$ and $\mathcal{L}^{3} \leq q \leq \mathcal{L}^{6}$,

$$
\begin{aligned}
\sum_{4} & \leq(0.11585+0.00365+0.24981+0.00003+0.00003) N \mathcal{L}^{-1} \\
& \leq 0.36938 N \mathcal{L}^{-1} .
\end{aligned}
$$

Now we estimate the $\sum_{5}$ in (5.20). Similarly to (5.3) and (5.4) we have

$$
\begin{aligned}
\sum_{5} \leq & \frac{16 N q^{1 / 2}}{\varphi(q)}\left\{(0.001 N)^{-1 / 2} \sum_{\chi(\bmod q)} \sum_{10^{4} \pi \leq|\gamma| \leq 10 \pi \mathcal{L}^{7} q^{-1}}|\gamma|^{-1 / 2}\right. \\
& +\left(\int_{1 / 2}^{59 / 60}+\int_{59 / 60}^{1}\right)(0.001 N)^{\alpha-1}(\log 0.001 N) \\
& \left.\times \sum_{\chi(\bmod q)} \sum_{\substack{10^{4} \pi \leq|\gamma| \leq 10 \pi \mathcal{L}^{7} q^{-1} \\
\beta \geq \alpha}}|\gamma|^{-1 / 2} d \alpha\right\}
\end{aligned}
$$


and for $\alpha \in[1 / 2,1)$,

$$
\begin{aligned}
& \sum_{\chi(\bmod q)} \sum_{\substack{10^{4} \pi \leq|\gamma| \leq 10 \pi \mathcal{L}^{7} q^{-1} \\
\beta \geq \alpha}}|\gamma|^{-1 / 2} \\
& \leq\left(10 \pi \mathcal{L}^{7} q^{-1}\right)^{-1 / 2} N\left(\alpha, q, 10 \pi \mathcal{L}^{7} q^{-1}\right)+\frac{1}{2} \int_{10^{4} \pi}^{10 \pi \mathcal{L}^{7} q^{-1}} y^{-3 / 2} N(\alpha, q, y) d y .
\end{aligned}
$$

By (2.4), (5.29) can be estimated further as $\leq 5 \varphi(q) q^{-1 / 2} \mathcal{L}^{3.5} \log \left(10 \pi \mathcal{L}^{7}\right)$. Hence the first term and the first integral in the curly brackets in (5.28) contribute to the right hand side of (5.28) at most

$$
\begin{aligned}
\leq & 16 \cdot 5 N \mathcal{L}^{3.5}\left(\log \left(10 \pi \mathcal{L}^{7}\right)\right) \\
& \times\left((0.001 N)^{-1 / 2}+\int_{1 / 2}^{59 / 60}(0.001 N)^{\alpha-1} \log 0.001 N d \alpha\right) \\
\leq & 0.0011 N \mathcal{L}^{-1} .
\end{aligned}
$$

From (5.28) to (5.30), it can be derived that

$$
\begin{aligned}
\sum_{5} \leq & 0.0011 N \mathcal{L}^{-1} \\
& +\frac{16 N q^{1 / 2}}{\varphi(q)}\left(10 \pi \mathcal{L}^{7} q^{-1}\right)^{-1 / 2} \\
& \times \int_{59 / 60}^{1}(0.001 N)^{\alpha-1}(\log 0.001 N) N\left(\alpha, q, 10 \pi \mathcal{L}^{7} q^{-1}\right) d \alpha \\
& +\frac{8 N q^{1 / 2}}{\varphi(q)} \int_{10 \pi \mathcal{L}^{7} q^{-1}}^{10} y^{-3 / 2} \\
& \times \int_{59 / 60}^{1}(0.001 N)^{\alpha-1}(\log 0.001 N) N(\alpha, q, y) d \alpha d y .
\end{aligned}
$$

Note that the integral $\int_{59 / 60}^{1}$ can be separated into

$$
\int_{59 / 60}^{1-0.478 / \log \left(10 \pi \mathcal{L}^{7}\right)}+\int_{1-0.478 / \log \left(10 \pi \mathcal{L}^{7}\right)}^{1}
$$

and that $10 \pi \mathcal{L}^{7} q^{-1} \geq 10^{4} \log q$ since $\mathcal{L}^{3} \leq q \leq \mathcal{L}^{6}$. Thus similarly to the treatment for (5.6) if we use [LW, Theorem 7] and the bound 14000 to estimate $N\left(\alpha, q, 10 \pi \mathcal{L}^{7} q^{-1}\right)$, the second term on the right hand side of (5.31) 
is

$$
\leq\left(0.02198+0.00046+5.26 \cdot 10^{-6}\right) N \mathcal{L}^{-1} \leq 0.02245 N \mathcal{L}^{-1}
$$

Now we estimate the last term on the right hand side of (5.31). Using (5.8), this term is

$$
\begin{aligned}
= & \frac{8 N q^{1 / 2}}{\varphi(q)} \int_{10^{4} \pi}^{10 \pi \mathcal{L}^{7} q^{-1}} y^{-3 / 2} \\
& \times \int_{59 / 60}^{1-0.478 / \log q y}(0.001 N)^{\alpha-1}(\log 0.001 N) N(\alpha, q, y) d \alpha d y \\
& +\frac{8 N q^{1 / 2}}{\varphi(q)} \int^{10 \pi \mathcal{L}^{7} q^{-1}} y^{-3 / 2} \\
& \times \int_{1-1 /\left(c_{1} \log q y\right)} \int^{1-0.478 / \log q y}(0.001 N)^{\alpha-1}(\log 0.001 N) N(\alpha, q, y) d \alpha d y \\
= & M_{3}+M_{4} \cdot
\end{aligned}
$$

The estimate for $M_{4}$ is very similar to that for $M_{2}$ defined as in (5.14); the difference is that the $\nu\left(\mathcal{L}^{3}\right)$ in $(5.16)$ and $(5.17)$ should be replaced by $\nu\left(\mathcal{L}^{6}\right)$ since the upper bound for $q$ is now $\mathcal{L}^{6}$. So by the bounds in (5.16) and (5.17) we get

$$
M_{4} \leq\left(2.1 \cdot 10^{-5}+2.4 \cdot 10^{-5}\right) N \mathcal{L}^{-1} \frac{\nu\left(3100^{6}\right)}{\nu\left(3100^{3}\right)} \leq 0.00006 N \mathcal{L}^{-1}
$$

For the estimation of $M_{3}$, we first decompose the integral $\int_{10^{4} \pi}^{10 \pi \mathcal{L}^{7} q^{-1}}$ as $\int_{10^{4} \pi}^{10^{4} \log q}+\int_{10^{4} \log q}^{10 \pi \mathcal{L}^{7} q^{-1}}$, and denote their contributions to $M_{3}$ by $M_{31}$ and $M_{32}$ respectively. Using [LW, Theorem 7] to bound $N\left(\alpha, q, 10^{4} \log q\right)$ and then Mathematica, we get

$$
\begin{aligned}
M_{31} \leq & N \mathcal{L}^{-1} \max _{\substack{3 \leq x \leq 6 \\
\mathcal{L} \geq 3100}}\left\{16\left(10^{4} \pi\right)^{-1 / 2} \nu\left(\mathcal{L}^{x}\right) e^{-\frac{0.478(\mathcal{L}+\log 0.001)}{\log \left(10^{4} \mathcal{L}^{x} \log \mathcal{L}^{x}\right)}}\right. \\
& \times\left(10^{16 / 60} \mathcal{L}^{1-9 x / 20}\left(\frac{254231}{\log \left(10^{4} \mathcal{L}^{x} \log \mathcal{L}^{x}\right)}+17102\right)\right. \\
& \times(x \log \mathcal{L})^{1 / 15} \log ^{6}\left(10^{4} \mathcal{L}^{x} \log \mathcal{L}^{x}\right) \\
& \left.\left.+16541 \mathcal{L}^{1-x / 2} \log ^{6}\left(10^{4} \log \mathcal{L}^{x}\right)\right)\right\} \\
\leq & 0.07227 N \mathcal{L}^{-1} .
\end{aligned}
$$


For the estimation of $M_{32}$, similarly to (5.9) and (5.10), since $\mathcal{L}^{3} \leq q \leq \mathcal{L}^{6}$, and then using Mathematica, we have

$$
\begin{aligned}
M_{32} \leq & N \mathcal{L}^{-1}\left\{8 e^{4 \cdot 0.478} \nu\left(\mathcal{L}^{6}\right) \mathcal{L}\right. \\
& \times \int_{\log \left(10^{4} \mathcal{L}^{3} \log \mathcal{L}^{3}\right)}^{\log \left(10 \pi \mathcal{L}^{7}\right)}(254231 / y+33643) \\
& \left.\times \frac{y^{6}(\mathcal{L}+\log 0.001)}{\mathcal{L}-4 y+\log 0.001} e^{-\frac{y}{2}-\frac{0.478(\mathcal{L}+\log 0.001)}{y}} d y\right\} \\
\leq & 0.03775 N \mathcal{L}^{-1} .
\end{aligned}
$$

(5.34)-(5.36) show that $(5.33)$ is $\leq(0.07227+0.03775+0.00006) N \mathcal{L}^{-1}$. This together with (5.32) and (5.31) ensures that

$$
\begin{aligned}
\sum_{5} & \leq(0.0011+0.02245+0.07227+0.03775+0.00006) N \mathcal{L}^{-1} \\
& \leq 0.1337 N \mathcal{L}^{-1} .
\end{aligned}
$$

Finally we estimate the $\sum_{6}$ in (5.20). We have

$$
\begin{aligned}
& \sum_{6} \leq \frac{5 q^{1 / 2} N}{\varphi(q)}\left\{N^{-1 / 2} \sum_{\chi(\bmod q)} \sum_{10 \pi \mathcal{L}^{7} q^{-1} \leq|\gamma| \leq T}|\gamma|^{-1}\right. \\
& \left.+\left(\int_{1 / 2}^{59 / 60}+\int_{59 / 60}^{1}\right) N^{\alpha-1}(\log N) \sum_{\chi(\bmod q)} \sum_{\substack{10 \pi \mathcal{L}^{7} q^{-1} \leq|\gamma| \leq T \\
\beta \geq \alpha}}|\gamma|^{-1} d \alpha\right\} .
\end{aligned}
$$

For any $\alpha \in[1 / 2,1)$ we have

$$
\begin{aligned}
& \sum_{\chi(\bmod q)} \sum_{\substack{10 \pi \mathcal{L}^{7} q^{-1} \leq|\gamma| \leq T \\
\beta \geq \alpha}}|\gamma|^{-1} \\
& \quad \leq T^{-1} N(\alpha, q, T)+\int_{10 \pi \mathcal{L}^{7} q^{-1}}^{T} y^{-2} N(\alpha, q, y) d y .
\end{aligned}
$$

We can use (2.4) to write (5.39) further as $\leq 101 \varphi(q) \log ^{2} \mathcal{L}$ on noting $\mathcal{L}^{3} \leq$ $q \leq \mathcal{L}^{6}, \mathcal{L} \geq 3100$. Thus similarly to (5.30) the first term and the first integral in the curly brackets in (5.38) contribute to (5.38) at most

$$
\leq 5 \cdot 101 N q^{1 / 2}(\log \mathcal{L})^{2}\left(N^{-1 / 2}+\int_{1 / 2}^{59 / 60} N^{\alpha-1} \log N d \alpha\right) \leq 0.00012 N \mathcal{L}^{-1}
$$


This together with (5.38) and (5.39) ensures that

$$
\begin{aligned}
\sum_{6} \leq & 0.00012 N \mathcal{L}^{-1}+\frac{5 N q^{1 / 2}}{\varphi(q) T} \int_{59 / 60}^{1} N^{\alpha-1}(\log N) N(\alpha, q, T) d \alpha \\
& +\frac{5 N q^{1 / 2}}{\varphi(q)} \int_{10 \pi \mathcal{L}^{7} q^{-1}}^{T} y^{-2} \int_{59 / 60}^{1} N^{\alpha-1}(\log N) N(\alpha, q, y) d \alpha d y
\end{aligned}
$$

In view of $\mathcal{L}^{3} \leq q \leq \mathcal{L}^{6}$ and $T=\mathcal{L}^{15}$, by [LW, Theorem 7], for $\alpha \in[59 / 60,1)$ we have

$$
N(\alpha, q, T) \leq(33643+254231 /(21 \log \mathcal{L}))(21 \log \mathcal{L})^{6} \mathcal{L}^{78 / 60} .
$$

Thus the second term on the right hand side of (5.40) is

$$
\begin{aligned}
& \leq 5 N T^{-1} q^{1 / 2} \varphi(q)^{-1}(33643+254231 /(21 \log \mathcal{L}))(21 \log \mathcal{L})^{6} \mathcal{L}^{78 / 60} \\
& \leq 7 \cdot 10^{-31} N \mathcal{L}^{-1} .
\end{aligned}
$$

To estimate the last term on the right hand side of (5.40), by (5.8) we first write the integral $\int_{59 / 60}^{1}$ as

$$
\int_{59 / 60}^{1-0.478 / \log q y}+\int_{1-0.478 / \log q y}^{1-0.10367089 / \log q y},
$$

and let $M_{5}$ and $M_{6}$ denote the contributions to (5.40) from the first and the second integrals in (5.42) respectively. Then by (5.40) and (5.41) we get

$$
\sum_{6} \leq\left(0.00012+7 \cdot 10^{-31}\right) N \mathcal{L}^{-1}+M_{5}+M_{6} .
$$

For $M_{6}$, we can use the bound $7000 \cdot 2$ in [LW, Table 5] to estimate $N(\alpha, q, y)$; so by Mathematica,

$$
\begin{aligned}
M_{6} & \leq N \mathcal{L}^{-1}\left\{70000 \mathcal{L}^{4} \nu\left(\mathcal{L}^{6}\right) \int_{\log \left(10 \pi \mathcal{L}^{7}\right)}^{\log \left(\mathcal{L}^{21}\right)} e^{-y-0.10367089 \mathcal{L} / y} d y\right\} \\
& \leq 6 \cdot 10^{-9} N \mathcal{L}^{-1} .
\end{aligned}
$$

For $M_{5}$, in view of $y \geq 10 \pi \mathcal{L}^{7} q^{-1} \geq 10^{4} \log q$, we can use [LW, Theorem 7 ] to get $N(\alpha, q, y) \leq(33643+254231 / \log q y)\left(q^{3} y^{4}\right)^{1 / 60}(\log q y)^{6}$. Thus

$$
\begin{aligned}
M_{5} \leq & N \mathcal{L}^{-1}\left\{5 \mathcal{L}^{1+6(1 / 2+1 / 20-1 / 15)} \nu\left(\mathcal{L}^{6}\right)\right. \\
& \left.\times \int_{\log \left(10 \pi \mathcal{L}^{7}\right)}^{21 \log \mathcal{L}}(33643+254231 / y) y^{6} e^{-14 y / 15-0.478 \mathcal{L} / y} d y\right\} .
\end{aligned}
$$

By Mathematica, the expression in the last curly brackets, as a function of $\mathcal{L}$, is shown to take supremum at $\mathcal{L}=3100$; and the supremum is $\leq 0.00007$. 
Thus we have $M_{5} \leq 0.00007 N \mathcal{L}^{-1}$. This together with (5.43) and (5.44) gives

$$
\sum_{6} \leq\left(0.00012+7 \cdot 10^{-31}+0.00007+6 \cdot 10^{-9}\right) N \mathcal{L}^{-1} \leq 0.0002 N \mathcal{L}^{-1} .
$$

This together with (5.20), (5.27) and (5.37) completes the proof of Lemma 5.2 .

6. Trigonometric sums over primes (II) and the proof of Theorem 1. In this section we first prove the following Proposition 6.1, which gives an explicit estimate for the $S(\alpha)$ defined by (3.8) for any real $\alpha$. We shall apply Proposition 6.1 to treat the integral over $\mathcal{M}_{4}$ defined as in (3.7) and then eventually complete the proof of Theorem 1. We remark that Proposition 6.1 is independent of the previous sections.

Proposition 6.1. Let $\alpha$ be a real number of the form

$$
\alpha=a / q+\theta / q^{2} \quad \text { with } q \geq 1,(a, q)=1,|\theta| \leq 1,
$$

and $S(\alpha)$ be defined as in (3.8) with 0.001 there replaced by any fixed real number $c$ satisfying $0<c<1$. Then for $N \geq 3$,

$$
|S(\alpha)| \leq 0.28 N q^{-0.5} \log ^{2} N+4 N^{0.8} \log ^{1.4} N+0.09 N^{0.5} q^{0.5} \log ^{2.5} N .
$$

By (3.6) of [RS2], we have $|S(\alpha)| \leq \sum_{n \leq N} \Lambda(n)<1.25506 N$; thus (6.2) holds if $1.25506 N \leq 4 N^{0.8} \log ^{1.4} N$, which enables us to assume that $N \geq$ $6.36 \cdot 10^{12}$. This together with [RS1, p. 265, (5.1) and (5.3)], implies $|S(\alpha)|$ $\leq 1.001102\left(N+N^{0.5}\right)+3 N^{1 / 3} \leq 1.0012 N$. Thus Proposition 6.1 holds if $q \leq(0.28 / 1.0012)^{2} \log ^{4} N$. Hence we may assume without loss of generality that $q>(0.28 / 1.0012)^{2} \log ^{4} N$. Also from $1.0012 N \leq 0.09 N^{0.5} q^{0.5} \log ^{2.5} N$ we get $q \geq(1.0012 / 0.09)^{2} N \log ^{-5} N$; and from $1.0012 N \leq 4 N^{0.8} \log ^{1.4} N$ we get $N \leq(4 / 1.0012)^{5} \log ^{7} N$. Thus from now on we can assume that

$$
(0.28 / 1.0012)^{2} \log ^{4} N<q<(1.0012 / 0.09)^{2} N \log ^{-5} N
$$

and

$$
N>(4 / 1.0012)^{5} \log ^{7} N .
$$

Note that by Mathematica, (6.4) and (6.3) imply

$$
N \geq \exp (30.95) \text { and } q \geq \exp (11) .
$$

Now we put

$$
U:=N^{2 / 5} \log ^{-4 / 5} N, \quad V:=N^{2 / 5} \log ^{1 / 5} N .
$$

Then the Vaughan identity (see, for example, [D, p. 138]) gives

$$
S(\alpha)=S_{1}(\alpha)+S_{2}(\alpha)+S_{3}(\alpha)+S_{4}(\alpha)
$$


where for $1 \leq j \leq 4$,

$$
S_{j}(\alpha)=\sum_{c N<n \leq N} \Lambda_{j}(n) e(\alpha n)
$$

with

$$
\begin{gathered}
\Lambda_{1}(n)=\left\{\begin{array}{ll}
\Lambda(n) & \text { if } n \leq U, \\
0 & \text { if } n>U,
\end{array} \quad \Lambda_{2}(n)=\sum_{m t=n, t \leq V} \mu(t) \log m,\right. \\
\Lambda_{3}(n)=-\sum_{(r t) \mid n, r \leq U, t \leq V} \Lambda(r) \mu(t), \quad \Lambda_{4}(n)=\sum_{m t=n, t>V} \mu(t) \sum_{r \mid m, r>U} \Lambda(r) .
\end{gathered}
$$

We now estimate $S_{j}(\alpha)$ for $1 \leq j \leq 4$ in the following Lemmas 6.2 and 6.5 to 6.7, and then complete the proof of Proposition 6.1.

Lemma 6.2. Let $S_{1}(\alpha)$ be defined as in (6.8). Then

$$
\left|S_{1}(\alpha)\right| \leq 1.02 N^{2 / 5} \log ^{-4 / 5} N \text {. }
$$

Proof. Note that $U \geq \exp (30.95 \cdot 2 / 5) 30.95^{-4 / 5} \geq \exp (9.634)$ by (6.5) and (6.6). The lemma follows from

$$
\left|S_{1}(\alpha)\right| \leq \sum_{n \leq U} \Lambda(n) \leq 1.001102\left(U+U^{0.5}\right)+3 U^{1 / 3}
$$

For the proof of Lemmas 6.5 to 6.7 below, we need the following auxiliary Lemmas 6.3 and 6.4.

Lemma 6.3. Suppose $X>0$ is a real number and $Y, Z$ are integers satisfying $Y \geq 1$. Let $\alpha$ be as in (6.1) and use $\|x\|$ to denote the fractional part of a real number $x$. Then

$$
\sum_{n=Z+1}^{Z+Y} \min \left(X, \frac{1}{2\|\alpha n\|}\right) \leq([Y / q]+1)(5 X+q \log q) .
$$

Proof. This is [WC, Lemma 2].

LEMMA 6.4. Under the notations of Lemma 6.3, if $X \geq 1$ and $q \geq 15$ then

$$
\sum_{n \leq X} \min \left(\frac{Y}{n}, \frac{1}{2\|\alpha n\|}\right) \leq 5 q+1.5 q \log q+X \log q+5 Y q^{-1} \log X .
$$

Proof. This is [WC, Lemma 3].

LEMMA 6.5. Let $S_{2}(\alpha)$ be defined as in (6.8) and $\alpha$ be given as in (6.1). We have

$$
\begin{aligned}
\left|S_{2}(\alpha)\right| \leq & 0.0282 N q^{-1 / 2} \log ^{2} N+0.0001 N^{4 / 5} \log ^{1.4} N \\
& +0.0006 N^{1 / 2} q^{1 / 2} \log ^{2.5} N
\end{aligned}
$$


Proof. By partial summation we have

$$
\left|S_{2}(\alpha)\right| \leq(\log N) \sum_{t \leq V} \min \left(\frac{N}{t}, \frac{1}{2\|\alpha t\|}\right) .
$$

By Lemma 6.4 and (6.6), this is

$$
\begin{aligned}
\leq\left(5 q+1.5 q \log q+N^{2 / 5}(\right. & \log N)^{1 / 5} \log q \\
& \left.+5 N q^{-1} \log \left(N^{2 / 5} \log ^{1 / 5} N\right)\right) \log N .
\end{aligned}
$$

Note that by the second inequality in (6.3) and $N \geq \exp (30.95)$ in (6.5) we have $\log q \leq \log N-12.34$. Thus $5 q+1.5 q \log q \leq 0.0006 N^{1 / 2} q^{1 / 2} \log ^{1.5} N$. By the first inequalities in (6.3) and (6.5) we get $5 N q^{-1} \log \left(N^{2 / 5} \log ^{1 / 5} N\right) \leq$ $0.0282 N q^{-1 / 2} \log N$, and $N^{2 / 5}(\log N)^{1 / 5} \log q \leq 0.0001 N^{4 / 5} \log ^{0.4} N$. These together with (6.9) complete the proof of Lemma 6.5.

Lemma 6.6. Let $S_{3}(\alpha)$ be defined as in (6.8) and $\alpha$ be given as in (6.1). Then

$$
\left|S_{3}(\alpha)\right| \leq 0.0131 N q^{-1 / 2} \log ^{2} N+0.8 N^{4 / 5} \log ^{1.4} N+0.0005 N^{1 / 2} q^{1 / 2} \log ^{2.5} N .
$$

Proof. Using $\sum_{r \mid m} \Lambda(r)=\log m \leq \log (U V)$ and Lemma 6.4 we get

$$
\left|S_{3}(\alpha)\right| \leq\left(5 q+1.5 q \log q+U V \log q+5 N q^{-1} \log (U V)\right) \log (U V) .
$$

By (6.6) and by $q \geq \exp (11)$ in (6.5) we have $(5 q+1.5 q \log q) \log (U V)$ $\leq 0.0005 N^{1 / 2} q^{1 / 2} \log ^{2.5} N, 5 N q^{-1} \log ^{2}(U V) \leq 0.0131 N q^{-1 / 2} \log ^{2} N$, and $U V(\log q) \log (U V) \leq 0.8 N^{4 / 5} \log ^{1.4} N$. Then the proof of Lemma 6.6 is complete.

Lemma 6.7. Let $S_{4}(\alpha)$ be defined as in (6.8) and $\alpha$ be given as in (6.1). Then

$$
\begin{aligned}
\left|S_{4}(\alpha)\right| \leq & 0.2264 N q^{-1 / 2} \log ^{2} N+2.787 N^{4 / 5} \log ^{1.4} N \\
& +0.0785 N^{1 / 2} q^{1 / 2} \log ^{2.5} N .
\end{aligned}
$$

Proof. Let

$$
\begin{gathered}
J=\left[\frac{\log (N /(U V))}{\log 2}+1\right], \quad a_{m}=\sum_{r \mid m, r>U} \Lambda(r), \\
M_{j}=2^{j-1} V \quad \text { for } 1 \leq j \leq J .
\end{gathered}
$$

Then by (6.8) and Cauchy's inequality we have

(6.11) $\left|S_{4}(\alpha)\right|$

$$
\leq \sum_{j=1}^{J}\left(\sum_{M_{j}<t \leq 2 M_{j}}|\mu(t)|\right)^{1 / 2}\left(\sum_{M_{j}<t \leq 2 M_{j}}\left|\sum_{\substack{c N / t<m \leq N / t \\ m>U}} a_{m} e(\alpha m t)\right|^{2}\right)^{1 / 2} .
$$


The expression in the last brackets in (6.11) is

$$
\leq \sum_{U<m \leq N / M_{j}} a_{m}^{2} \sum_{U<l \leq N / M_{j}} \min \left(M_{j}+0.5,(2\|\alpha(m-l)\|)^{-1}\right) .
$$

If we use Lemma 6.3 to estimate the last sum over $l$, the above is

$$
\leq\left(\left[\frac{\left[N / M_{j}\right]-[U]}{q}\right]+1\right)\left(5\left(M_{j}+0.5\right)+q \log q\right) \sum_{U<m \leq N / M_{j}} a_{m}^{2} .
$$

Denote by $W$ the sum over $m$ in (6.12). In view of the definition of $a_{m}$ we have

$$
W=\sum_{r, s>U} \Lambda(r) \Lambda(s) \#\left\{U<m \leq N / M_{j}: r, s \mid m\right\} .
$$

Now if $(r, s)=1$ then $r s \mid m$, whence

$$
U^{2}<r s \leq m \leq N / M_{j} \leq N / V .
$$

This is a contradiction, so that $r, s$ must have a common factor. It follows that

$$
W=\sum_{p^{e}, p^{f}>U} \log ^{2} p \#\left\{U<m \leq N / M_{j}: p^{\max (e, f)} \mid m\right\},
$$

so that

$$
\begin{aligned}
W \leq & N M_{j}^{-1} \sum_{U<p^{e}, p^{f} \leq N / M_{j}} p^{-\max (e, f)} \log ^{2} p \\
= & N M_{j}^{-1}\left\{\sum_{U<p \leq N / M_{j}} p^{-1} \log ^{2} p+\sum_{U<p^{e} \leq N / M_{j}, e \geq 2} p^{-e} \log ^{2} p\right. \\
& \left.+2 \sum_{U<p^{e}<p^{f} \leq N / M_{j}} p^{-f} \log ^{2} p\right\} \\
= & : N M_{j}^{-1}\left(W_{1}+W_{2}+2 W_{3}\right) .
\end{aligned}
$$

Clearly

$$
\begin{aligned}
W_{2} & =\sum_{2 \leq e \leq\left(\log N / M_{j}\right) / \log 2} \sum_{U^{1 / e}<p \leq\left(N / M_{j}\right)^{1 / e}} p^{-e} \log ^{2} p \\
& \leq\left(\log N / M_{j}\right)^{2} \sum_{2 \leq e \leq\left(\log N / M_{j}\right) / \log 2} e^{-2} \sum_{U^{1 / e}<p} p^{-e} .
\end{aligned}
$$

The last sum over $p$ is, in view of $e \geq 2$,

$$
\leq \int_{U^{1 / e}}^{\infty} x^{-e} d \sum_{n \leq x} 1 \leq e \int_{U^{1 / e}}^{\infty} x^{-e} d x \leq \frac{e}{e-1} U^{(1-e) / e} .
$$


This yields, on noting $U=N^{2 / 5} \log ^{-4 / 5} N, V=N^{2 / 5} \log ^{1 / 5} N$, and $N \geq$ $\exp (30.95)$,

$$
\begin{aligned}
W_{2} & \leq\left(\log N / M_{j}\right)^{2} \sum_{2 \leq e \leq\left(\log N / M_{j}\right) / \log 2} \frac{1}{e(e-1)} U^{(1-e) / e} \\
& \leq U^{-1 / 2}\left(\log N / M_{j}\right)^{2} \leq 2.5876 .
\end{aligned}
$$

For $W_{3}$ we have

$$
\begin{aligned}
W_{3} & =\sum_{2 \leq f \leq\left(\log N / M_{j}\right) / \log 2} \sum_{1 \leq e<f} \sum_{U^{1 / e}<p \leq\left(N / M_{j}\right)^{1 / 5}} p^{-f} \log ^{2} p \\
& \leq\left(\log N / M_{j}\right)^{2} \sum_{2 \leq f \leq\left(\log N / M_{j}\right) / \log 2} \frac{1}{f^{2}} \sum_{1 \leq e<f} \sum_{U^{1 / e}<p} p^{-f} .
\end{aligned}
$$

The last sum over $p$ is, in view of $f \geq 2$,

$$
\leq \sum_{n>U^{1 / e}} n^{-f}=\int_{U^{1 / e}}^{\infty} x^{-f} d \sum_{n \leq x} 1 \leq f \int_{U^{1 / e}}^{\infty} x^{-f} d x=\frac{f}{f-1} U^{(1-f) / e},
$$

so that, in view of $f-1 \geq e$,

$$
\begin{aligned}
W_{3} & \leq\left(\log N / M_{j}\right)^{2} \sum_{2 \leq f \leq\left(\log N / M_{j}\right) / \log 2} \frac{1}{f(f-1)} \sum_{1 \leq e<f} U^{(1-f) / e} \\
& \leq U^{-1}\left(\log N / M_{j}\right)^{2} \sum_{2 \leq f \leq\left(\log N / M_{j}\right) / \log 2} \frac{1}{f} \leq 0.0681 .
\end{aligned}
$$

To estimate $W_{1}$ we use Stieltjes integral. Then one has by integral by parts

$$
\begin{aligned}
W_{1}= & \left(\log N / M_{j}\right) \sum_{p \leq N / M_{j}} p^{-1} \log p-(\log U) \sum_{p \leq U} p^{-1} \log p \\
& -\int_{U}^{N / M_{j}} \frac{1}{x} \sum_{p \leq x} p^{-1} \log p d x .
\end{aligned}
$$

Note that the above $N / M_{j} \geq x \geq U \geq 15000$. Then Theorem 6 and (2.11) of [RS2] yield, for any $x \in\left[U, N / M_{j}\right]$,

$$
\log x-1.385 \leq \sum_{p \leq x} p^{-1} \log p \leq \log x-1.28 .
$$

This together with (6.16) produces

$$
W_{1} \leq \frac{1}{2}\left(\log ^{2}\left(N / M_{j}\right)-\log ^{2} U\right)+0.105 \log N / M_{j} .
$$


Now substituting (6.14), (6.15) and (6.17) into (6.13) we get

$$
\begin{aligned}
W \leq & \frac{1}{2} N M_{j}^{-1}\left(\log ^{2} N / M_{j}-\log ^{2} U+2 \cdot 0.105 \log N / M_{j}\right. \\
& +2 \cdot 2.5876+4 \cdot 0.0681) \\
\leq & 0.5203\left(1-((\log U) /(\log N / V))^{2}\right) N M_{j}^{-1} \log ^{2} N / V .
\end{aligned}
$$

Note that

$$
\frac{\log U}{\log N / V}=\frac{\frac{2}{5} \log N-\frac{4}{5} \log \log N}{\frac{3}{5} \log N-\frac{1}{5} \log \log N}=\frac{2-\frac{4 \log \log N}{\log N}}{3-\frac{\log \log N}{\log N}} \geq \frac{2}{3} .
$$

So the above gives

$$
W \leq 0.5203\left(1-\left(\frac{2}{3}\right)^{2}\right) N M_{j}^{-1} \log ^{2} N / V=0.5203(5 / 9) N M_{j}^{-1} \log ^{2} N / V .
$$

This enables us to estimate (6.12) further as

$$
\begin{aligned}
(6.18) \leq & 0.5203(5 / 9) N M_{j}^{-1}\left(\frac{N / M_{j}-U+1}{q}+1\right) \\
& \times\left(5 M_{j}+2.5+q \log q\right)\left(\log \frac{N}{V}\right)^{2} \\
\leq & 0.5203(5 / 9)\left(\frac{5 N}{q}+5 M_{j}+\frac{N}{M_{j}} \log q+q \log q+\left\{\frac{5 M_{j}}{q}-\frac{5 M_{j} U}{q}\right.\right. \\
& \left.\left.+\frac{2.5 N}{M_{j} q}+2.5-\frac{2.5 U}{q}-U \log q+\frac{2.5}{q}+\log q\right\}\right) \frac{N}{M_{j}}\left(\log \frac{N}{V}\right)^{2} .
\end{aligned}
$$

In view of $N \geq \exp (30.95), M_{j} \geq V$ and $U \geq \exp (9.634)$, the expression in the above curly brackets is $<0$. Thus (6.18) is

$$
\leq 0.5203(5 / 9)\left(\frac{5 N}{q}+5 M_{j}+\frac{N}{M_{j}} \log q+q \log q\right) \frac{N}{M_{j}}\left(\log \frac{N}{V}\right)^{2} .
$$

Again in view of $M_{j} \geq V \geq 30.95^{1 / 5} \exp (30.95 \cdot 2 / 5) \geq \exp (13.066)$, we have

$$
\sum_{M_{j}<t \leq 2 M_{j}}|\mu(t)|=\sum_{d^{2} \leq 2 M_{j}} \mu(d) \sum_{\substack{M_{j}<t \leq 2 M_{j} \\ d^{2} \mid t}} 1 \leq 0.6111 M_{j} .
$$

Substituting (6.19) and (6.20) into (6.11) we get

$$
\begin{aligned}
& \left|S_{4}(\alpha)\right| \\
\leq & \sqrt{0.6111 \cdot 0.5203(5 / 9)} N^{1 / 2}\left(\log \frac{N}{V}\right) \\
& \times \sum_{j=1}^{J}\left(\left(5 N q^{-1}\right)^{1 / 2}+\left(5 M_{j}\right)^{1 / 2}+\left(N M_{j}^{-1} \log q\right)^{1 / 2}+(q \log q)^{1 / 2}\right) .
\end{aligned}
$$


By (6.10) we have $\sum_{j=1}^{J} M_{j}^{1 / 2} \leq(2+\sqrt{2})(N / U)^{1 / 2}$, and $\sum_{j=1}^{J} M_{j}^{-1 / 2} \leq$ $(2+\sqrt{2}) V^{-1 / 2}$. Thus (6.21) can be estimated further as

$$
\begin{aligned}
\leq & \sqrt{5 \cdot 0.6111 \cdot 0.5203(5 / 9)} N q^{-1 / 2} \\
& \times(1+(\log (N /(U V))) / \log 2) \log \left(N V^{-1}\right) \\
& +(2+\sqrt{2}) \sqrt{5 \cdot 0.6111 \cdot 0.5203(5 / 9)} N U^{-1 / 2} \log \left(N V^{-1}\right) \\
& +(2+\sqrt{2}) \sqrt{0.6111 \cdot 0.5203(5 / 9)} N V^{-1 / 2}(\log q)^{1 / 2} \log \left(N V^{-1}\right) \\
& +\sqrt{0.6111 \cdot 0.5203(5 / 9)} N^{1 / 2} q^{1 / 2}(\log q)^{1 / 2} \\
& \times(1+(\log (N /(U V))) / \log 2) \log \left(N V^{-1}\right) .
\end{aligned}
$$

If we make use of (6.6) and $N \geq \exp (30.95)$ in (6.5), the four terms in (6.22) can be estimated as $\leq 0.2264 N q^{-1 / 2} \log ^{2} N, \leq 1.926 N^{4 / 5} \log ^{1.4} N$, $\leq 0.861 N^{4 / 5} \log ^{1.4} N$, and $\leq 0.0785 N^{1 / 2} q^{1 / 2} \log ^{2.5} N$ respectively. Then the proof of Lemma 6.7 is complete.

Proof of Proposition 6.1. Now (6.2) follows from (6.7) and Lemmas 6.2 and 6.5 to 6.7 .

Proof of Theorem 1. For any $\alpha$ given as in (3.2), by Proposition 6.1, $|S(\alpha)| \leq 0.28 N q^{-1 / 2} \mathcal{L}^{2}+4 N^{4 / 5} \mathcal{L}^{1.4}+0.09 N^{1 / 2} q^{1 / 2} \mathcal{L}^{2.5}$. Thus if $\alpha \in \mathcal{M}_{4}$, which is defined by (3.7), then in view of $\mathcal{L}^{6}=P_{1} \leq q \leq N \mathcal{L}^{-7}$ we get, for $\mathcal{L} \geq 3100$,

$$
\begin{aligned}
|S(\alpha)| \leq & \max \left\{0.28 N P_{1}^{-1 / 2} \mathcal{L}^{2}+4 N^{4 / 5} \mathcal{L}^{1.4}+0.09 N^{1 / 2} P_{1}^{1 / 2} \mathcal{L}^{2.5}\right. \\
& \left.0.28 N\left(N \mathcal{L}^{-7}\right)^{-1 / 2} \mathcal{L}^{2}+4 N^{4 / 5} \mathcal{L}^{1.4}+0.09 N^{1 / 2}\left(N \mathcal{L}^{-7}\right)^{1 / 2} \mathcal{L}^{2.5}\right\} \\
\leq & 0.3 N \mathcal{L}^{-1} .
\end{aligned}
$$

In combination with Lemmas 5.1 and 5.2 , this yields $|S(\alpha)| \leq 0.5033 N \mathcal{L}^{-1}$ for $\alpha \in \mathcal{M}_{2} \cup \mathcal{M}_{3} \cup \mathcal{M}_{4}$ and $\mathcal{L} \geq 3100$. Thus

$$
\left|I_{2}(N)+I_{3}(N)+I_{4}(N)\right| \leq 0.5033 N \mathcal{L}^{-1} \int_{0}^{1}|S(\alpha)|^{2} d \alpha \leq 0.5033 N \sum_{p \leq N} \log p .
$$

By [RS1, Theorem 6, (5.1)], the last sum over $p$ is $<1.001102 N$. Thus the above is $\leq 0.5033 \cdot 1.001102 N^{2} \leq 0.51 N^{2}$. This together with (3.12) and Lemma 4.3 gives, for $\mathcal{L} \geq 3100$,

$$
I(N) \geq(0.5437-0.51) N^{2}-3 N^{1.5} \mathcal{L}^{3} \geq 0.03 N^{2} .
$$

In view of (3.9) the proof of Theorem 1 is complete.

Acknowledgements. The authors would like to express their thanks to the referee for his valuable comments on the improvement of Lemma 6.7. 


\section{Errata to $[\mathrm{LW}]$ :}

- Page 278, line -2 : Replace 4 and 364 by 2 and 182 respectively.

- Page 284, lines -15 and -7: Replace Lemma 3.6 by Lemma 3.2.

\section{References}

[B] K. G. Borozdkin, On I. M. Vinogradov's constant, in: Proc. 3rd All-Union Math. Conf., Izdat. Akad. Nauk SSSR, Moscow, 1 (1956), 3 (in Russian).

[CW] J. R. Chen and T. Z. Wang, On odd Goldbach problem, Acta Math. Sinica 32 (1989), 702-718.

[D] H. Davenport, Multiplicative Number Theory, 2nd ed., Grad. Texts in Math. 74, Springer, 1980.

[DERZ] J. M. Deshouillers, G. Effinger, H. te Riele and D. Zinoviev, A complete Vinogradov 3-primes theorem under the Riemann hypothesis, Electron. Res. Announc. Amer. Math. Soc. 3 (1997), 99-104.

[HR] H. Halberstam and H.-E. Richert, Sieve Methods, Academic Press, 1974.

[HL] G. H. Hardy and J. E. Littlewood, Some problems of "Partitio Numerorum", III: On the expression of a number as a sum of primes, Acta Math. 44 (1923), $1-70$.

[LT] M. C. Liu and K. M. Tsang, Small prime solutions of linear equations, in: Théorie des Nombres, J.-M. De Koninck and C. Levesque (eds.), Walter de Gruyter, 1989, 595-624.

[LW] M. C. Liu and T. Z. Wang, Distribution of zeros of Dirichlet L-functions and an explicit formula for $\psi(t, \chi)$, Acta Arith. 102 (2002), 261-293.

[RS1] J. B. Rosser and L. Schoenfeld, Sharper bounds for the Chebyshev functions $\theta(x)$ and $\psi(x)$, Math. Comp. 29 (1975), 243-269.

[RS2] - - - Approximate formulas for some functions of prime numbers, Illinois J. Math. 6 (1962), 64-94.

[S] Y. Saouter, Checking the odd Goldbach conjecture up to $10^{20}$, Math. Comp. 67 (1998), 863-866.

[T] E. C. Titchmarsh, The Theory of the Riemann Zeta-Function, 2nd ed., Oxford Univ. Press, 1986.

[V] I. M. Vinogradov, Representation of an odd number as a sum of three primes, Dokl. Akad. Nauk SSSR 15 (1937), No. 6-7, 291-294.

[WC] T. Z. Wang and J. R. Chen, An estimate for linear exponential sums over primes, Acta Math. Sinica 37 (1994), 25-31.

Department of Mathematics

The University of Hong Kong

Pokfulam, Hong Kong

Current address:

P.O. Box 625

Alhambra, CA 91802, U.S.A.

E-mail: matmcliu@yahoo.com
Department of Mathematics Henan University Kaifeng 475001, Henan P.R. China E-mail: wangtz@henu.edu.cn

Received on 29.8.2000

and in revised form on 26.9.2001 